\title{
A Physically Inspired Equivalent Neural Network Circuit Model for SoC Estimation of Electrochemical Cells
}

\author{
Stefano Leonori *(D), Luca Baldini (D), Antonello Rizzi (D) and Fabio Massimo Frattale Mascioli
}

check for updates

Citation: Leonori, S.; Baldini, L.; Rizzi, A.; Frattale Mascioli, F.M. A Physically Inspired Equivalent Neural Network Circuit Model for SoC Estimation of Electrochemical Cells. Energies 2021, 14, 7386. https://doi.org/10.3390/en14217386

Academic Editor: Cai Shen

Received: 1 October 2021

Accepted: 2 November 2021

Published: 5 November 2021

Publisher's Note: MDPI stays neutral with regard to jurisdictional claims in published maps and institutional affiliations.

Copyright: (c) 2021 by the authors. Licensee MDPI, Basel, Switzerland. This article is an open access article distributed under the terms and conditions of the Creative Commons Attribution (CC BY) license (https:// creativecommons.org/licenses/by/ $4.0 /)$.
Department of Information Engineering, Electronics and Telecommunications, University of Rome "La Sapienza", Via Eudossiana 18, 00184 Rome, Italy; luca.baldini@uniroma1.it (L.B.); antonello.rizzi@uniroma1.it (A.R.); fabiomassimo.frattalemascioli@uniroma1.it (F.M.F.M.)

* Correspondence: stefano.leonori@uniroma1.it

\begin{abstract}
Battery Management System (BMS) design for Lithium-ion batteries State of Charge (SoC) prediction has a crucial role in Electric Vehicles (EVs) and smart grids development. The need to design compact, light and fast devices requires finding a suitable trade-off between effectiveness and efficiency. In the literature, it is well emphasized that the application of electrochemical-based methods such as the Pseudo-Two-Dimensional (P2D) model is computationally prohibitive and requires significant simplifications. Conversely, plain Equivalent Circuit Models (ECM) are too simple and unable to represent the cell dynamics. The application of an Ensemble Neural Network (ENN) as Equivalent Neural Network Circuit (ENNC) emerged as a promising solution able to synthesize expressive and computationally efficient models. Indeed, with the support of a suitable dataset, an ENN can be configured to represent a given ECM, modeling each lumped parameter through an assigned Neural Network (NN). Accordingly, the ENNC system is able to keep a physical description of the battery cell while approximating the non-linear dynamic of each component. The paper proposes a novel ENNC battery named Physical Inspired-Equivalent Neural Network Circuit (PI-ENNC) whose ensemble architecture relies on a fractional-order Extended Single Particle (ESP) Lithium-ion cell formulation. The PI-ENNC is designed to approximate the ESP transfer functions referred to the ohmic effects, the electrolyte diffusion and the non-uniform charge distribution in the cell. The proposed model has been tested with three publicly available datasets, investigating the model behavior according to two different training strategies and with different input configurations. In order to prove its effectiveness, results have been compared with a simpler version proposed in a previous work. Results highlight the effectiveness of PI-ENNC in SoC prediction, underlining the importance of designing an ENN architecture that leverages on equations and constraints that reflect the physical phenomena of the cell.
\end{abstract}

Keywords: battery management system; neural networks; Li-ion battery; ensemble neural network; circuit equivalent models; state of charge; prediction; machine learning; data driven

\section{Introduction}

Li-Ion batteries emerge as the main Energy Storage System (ESS) technology in the automotive field and smart grids for the integration of renewable energy sources, electric mobility, Vehicle to Grid (V2G) systems and demand response programs into the electric grid [1-4]. With respect to other technologies, Lithium-Ion batteries are characterized by high energy densities, fast-response and competitive costs. In [2,5], the authors reported a detailed review on the Lithium-Ion ESS applications in automotive and V2G systems, highlighting the increasing efforts in the research field as well as all the progress in energy management, safety issues and cost abatement. Concerning the latter, Lithium-Ion batteries costs have been reduced by $70 \%$ in the last decade. Although the price is still considerably high ("500 (\$/kWh) that is about USD 35,000 to 40,000 of the overall EVs' price" [2]), it is expected to reach values lower than $200(\$ / \mathrm{kWh})[6]$. 
In addition to possible improvements in chemical materials, cell design and circuital components, the research activity directions move towards the optimization of intelligent Battery Management System (BMS) devices to improve the reliability of the battery pack. The BMS is in charge of monitoring and managing every cell inside the battery system ensuring that their working points are bounded within the safety area [7]. Moreover, the excessive charging/discharging currents and power transients should be monitored by the BMS avoiding possible damages and deterioration of the battery cell. Usually, its working frequency is equal or greater than one second and its hardware must be as light and compact as possible in order to fit into an Electric Vehicle (EV) [8]. A detailed study on the hardware design, functioning and BMS architecture is discussed in $[7,9]$.

Designing an effective and efficient prediction model able to approximate the cell dynamic and thus its State of Charge (SoC) is a crucial facet for BMS systems, as widely emphasized in the literature [10-12]. Indeed, other than maximizing the prediction effectiveness, the BMS algorithm should be characterized by low computational costs.

Amongst the prediction models proposed in the literature, direct methods emerged as the most popular approaches due to their intuitive reasoning and ease of implementation. Indeed, they are only based on "Direct measures of physical battery properties such as voltage, current, and temperature, and then, by using an equation or relationship" [13]. Typical examples of direct methods are Coulomb Counting (CC) estimation [14], the Open Circuit Voltage (OCV) estimation and the Electrochemical Impedance Spectroscopy (EIS) estimation $[13,15,16]$. By leveraging on a limited number of equations, relations and direct measures, these methods appear very efficient in terms of computational cost and ease of implementation.

However, SoC estimation techniques based on direct measures, i.e., OCV methods and CC algorithms, are not satisfactory for the battery pack installation in complex power systems like automotive systems and smart grids which usually show high power peaks and power transients. Indeed, these models are not able to take into account the ohmic and the electrolyte diffusion effects in the cell, which produce very high uncertainties during power transients. This phenomenon is well described in [17] by means of a mechanical analogy. In the manuscript, the problem of estimating the amount of charge stored in a cell based on voltage and current measurements has been associated with the problem of estimating the volume of the water stored in a reservoir based on pressure and flow rate measurements.

Even though CC is widely adopted $[3,18,19]$, in $[20,21]$, the authors described four types of error sources that can compromise the accuracy of the SoC prediction. These can be summarized in: (i) current measurement error; (ii) error due to the approximation of current integration with respect to time; (iii) uncertainty in the knowledge of battery capacity; and the (iv) error (drift) in timing oscillator.

Considering the same approach, three types of error are summarized also for voltagebased (or OCV-based) models [21]: (i) OCV-SOC modeling error; (ii) voltage-drop modeling error; (iii) voltage measurement error.

For the motivations discussed above, the authors suggest the implementation of suitable filters supporting the prediction model to improve the SoC estimation. Non-linear Kalman Filters (KFs) such as Extended Kalman Filter (EKF) [22] and Unscented Kalman Filter (UKF) [23] emerge as interesting and widely adopted solutions for this purpose as well as Recursive Least Square, Particle and H-infinity filters [11,13].

In order to design more effective BMSs, non-linear KFs are usually supported with accurate prediction models impacting on the computational costs of the procedure as discussed in [24-28].

In the literature, there are several surveys and reviews concerning the categorization of BMS prediction models. In [9], the authors distinguished three main approaches: electrochemical models, Equivalent Circuit Models (ECMs) and black box models. A similar categorization is proposed in $[11,29]$, where authors summarize the SoC estimation methods into direct measures, computational intelligence and model-based approaches [11], 
whereas in [29], they are distinguished by electrochemical, empirical-ECM and empiricalNeural Networks (NNs).

Electrochemical models are based on high-order differential equations representing the chemical and electrochemical kinetics, as well as the mechanical and transport laws used to simulate the Li-ion battery's characteristics and reactions. These models can accurately predict physical quantities through a fine tuning of the model coefficients and parameters. However, they are usually characterized by considerably high computational costs. Indeed, electrochemical models consist in high order differential equations, such as Pseudo-two-Dimensional (P2D) models [30], which are not suitable for online applications. For this reason, P2D models are often reformulated in order to provide a good trade-off between the prediction accuracy and computational costs [31-35].

Conversely, ECMs are featured by fewer (lumped) parameters and they are often used in online applications. Indeed, these models are linear (or piece-wise linear) systems that can be represented by an equivalent electric circuit. However, in most cases they lack physical interpretation and can only provide the description of high-level physical quantities [12]. In many studies, ECMs models have been tuned offline according to a suitable optimization algorithm since the real lumped parameter values are hardly ever known a priori. Evolutionary algorithms and Swarm Optimization approaches such as Particle Swarm Optimization (PSO) have been proved to be effective solutions for correctly determining meaningful values for the ECM parameters [36-40].

Regarding black box models, they describe a linear or non-linear mapping function of battery voltage response characteristics [9]. According to [11,29], these models rely on machine learning algorithms and data driven techniques. Therefore, they neglect the internal mechanisms of the cell and do not provide further physical explanation of the cell behavior since the model is learned by considering only the dataset at hand. Concerning the models proposed in the literature, Fuzzy Inference Systems (FISs) [41,42], NNs [43-47] and Support Vector Machines (SVMs) $[48,49]$ have been widely explored.

Beyond the categorizations written above, it is interesting to highlight the following points. In the case of an electrochemical model, a suitable simplification and problem linearization can collapse its transfer functions into an ECM representation, that is, when the differential equations are simplified (i.e., truncated) to the first-order as in [16,34]. An ECM can be synthesized through a suitable machine learning model, (e.g., NNs, SVMs or FISs) in order to tune the lumped parameters non-linearities according to the available dataset representing the cell for approximation. This approach would be able to provide some degree of interpretability of the ECM moving toward a white box equivalent model $[9,46]$.

As proposed in [46], the application of Equivalent Neural Network Circuits (ENNCs) based on Ensemble Neural Network (ENN) systems [50] is an interesting solution. Indeed, a given ENN can be configured to reproduce a corresponding ECM by assigning each NN of the ensemble system to an ECM lumped parameter in order to better approximate the circuit non-linearities and therefore the cell behavior. In this paper, a Physical Inspired Equivalent Neural Network Circuit (PI-ENNC) is proposed as an extension of the ENNC introduced in [46]. The ENN architecture of PI-ENNC has been conceived to attain a strong analogy with the ESP fractional-order model proposed in [34]. The tests are focused on an exhaustive comparison between the ENNC and the PI-ENNC considering the SoC prediction and different training procedure and dataset.

The main contributions of this work can be summarized as follows:

- An exhaustive description of the ENNC model and its representation of a corresponding ECM;

- The introduction of a novel physical-inspired model where the NN architecture approximates the ESP transfer functions referred to as the ohmic effects, the electrolyte diffusion and the non-uniform charge distribution in the cell;

- A comparison between the PI-ENNC model and its basic version using different training and test datasets available in the literature as well as different training procedures. 
The paper is organized as follows: in Section 2 the BMS problem formulation is introduced; in Section 3 the BMS functioning is completely described explaining the KF application and its configuration settings; in Section 4, an in-depth description of the EMC and ENNC models are provided; Section 5 is dedicated to the PI-ENNC, which is the object of this paper. The manuscript continues with the dataset presentation, test settings and results commented in Sections 6-8, respectively. Finally, conclusions are drawn in Section 9. In Appendix A, additional figures regarding the predicted SoC and output voltage time series are provided.

\section{Problem Formulation}

An electrochemical cell can be formulated as a non-linear dynamic system described by the state equations:

$$
\begin{aligned}
& \mathbf{x}[k+1]=\mathcal{F}(\mathbf{x}[k], \mathbf{u}[k]) \\
& \mathbf{y}[k+1]=\mathcal{G}(\mathbf{x}[k], \mathbf{u}[k])
\end{aligned}
$$

where $\mathbf{x}, \mathbf{u}$ and $\mathbf{y}$ are respectively the cell state vector, the input and the output array and $\mathcal{F}$ and $\mathcal{G}$ are both non-linear functions. Equation (1) determines the cell state evolution whilst Equation (2) evaluates the output of the dynamic system.

As reported in many studies focused on the BMS generation through circuital models [8,51], the main physical quantities that contribute to the electrochemical cell state are the input current, the output voltage, the cell temperature and the State of Charge (SoC). According to [17], the terminal voltage of an electrochemical cell can be summarized as the superposition of three contributions:

- Quasi-stationary voltage $V_{q s t}$ : it corresponds to the OCV curve representing the voltage contribution related to the amount of charge actually stored in the cell. The use of the suffix quasi-stationary instead of OCV aims at distinguishing the manufacturer curve from the voltage contribution to predict while the cell is working, i.e. when the circuit is closed.

- Dynamic voltage contribution $V_{d y n}$ : this contribution takes into account the voltage transient response due to the electrolyte diffusion phenomenon whose effects can be approximated to a low-pass filtering with respect to the input current [34].

- Instantaneous voltage contribution $V_{\text {ist }}$ : it takes into account the internal resistance, namely the ohmic effects and the electrochemical kinetics of the cell as well [34]. Due to its nature, this contribution does not depend on previous battery states, i.e., it has no memory and it is mainly a function of the current.

According to the previous discussion, the terminal voltage of a battery cell can be formulated as follows:

$$
V_{\text {out }}[k]=V_{\text {qst }}[k]+V_{d y n}[k]+V_{\text {ist }}[k]
$$

Due to the non-linear electrochemical processes occurring during the charging/discharging phases, $V_{d y n}, V_{q s t}$ and $V_{i s t}$ may show non-linear dependencies with respect to the main physical quantities, i.e., the input current $I_{i n}$, the state of charge $S o C$ and the cell temperature $T_{\text {in }}$ :

$$
\begin{gathered}
V_{d y n}[k]=\mathcal{F}_{d y n}\left(V_{d y n}[k-1], \operatorname{SoC}[k], I_{i n}[k], T_{i n}[k]\right) \\
V_{i s t}[k]=\mathcal{V}_{i s t}\left(T_{i n}[k], I_{i n}[k], \operatorname{SoC}[k]\right)=\mathcal{R}_{\text {ist }}\left(T_{\text {in }}[k], I_{\text {in }}[k]\right) I_{\text {in }}[k] \\
V_{q s t}[k]=\mathcal{V}_{q s t}(\operatorname{SoC}[k])
\end{gathered}
$$

where $k$ is the time slot index.

In Equation (4), $\mathcal{F}_{d y n}$ addressed the non-linearities involved in the dynamic voltage contribution. As stated before, $\mathcal{F}_{d y n}$ has a low-pass filter behavior with respect to the input current $I_{i n}$. In Equation (5), $\mathcal{R}_{\text {ist }}$ represents the resistance function that models the instantaneous voltage $V_{i s t}$. It is supposed to approximate the ohmic effects involved in the cell, does not have memory and is only a function of the load current and the temperature. 
Finally, $\mathcal{V}_{q s t}$ approximates the OCV curve, and hence, it is supposed to be only a function of the $\mathrm{SoC}$, as shown in Equation (6).

Regarding the SoC variable estimation, it is defined as follows:

$$
\operatorname{SoC}[k]=\mathcal{F}_{\text {SoC }}\left(\operatorname{SoC}[k-1], I_{\text {in }}[k]\right)
$$

where $\mathcal{F}_{S o C}$ is calculated by means of a normalized discrete approximation of the load current integral, known as the CC algorithm [14]:

$$
\mathcal{F}_{\text {SoC }}\left(\operatorname{SoC}[k], I_{\text {in }}[k]\right)=S o C[k]+\frac{T_{S}}{C_{n}} I_{\text {in }}[k]
$$

In Equation (8), $C_{n}$ is the cell capacity that is expressed in $[\mathrm{Ah}]$ whilst $T_{S}$ is the time length sample.

In Figure 1, a schematic diagram of the BMS model is shown according to the problem formulation just discussed. $I_{i n}, T_{i n}$ and $S o C$ undergoes a preprocessing stage in the Input Mapping block. Specifically, this block performs a linear transformation, which normalizes $I_{i n}, T_{i n}$ and $S o C$ in the $[0,1]$ range according to their respective maximum and minimum values observed in the training set. Additionally, the input quantities are collected in $\mathbf{u}_{d y n}$, $\mathbf{u}_{i s t}$ and $\mathbf{u}_{q s t}$, which serves as input for the blocks that implement Equations (4)-(6), i.e., the main voltage contributions that determine the output voltage $V_{\text {out }}$ :

$$
\begin{array}{r}
\mathbf{u}_{d y n}=\left[S o C, I_{i n}, T_{i n}\right] \\
\mathbf{u}_{i s t}=\left[I_{i n}, T_{i n}, S o C\right] \\
\mathbf{u}_{q s t}=\left[S o C, I_{i n}\right]
\end{array}
$$

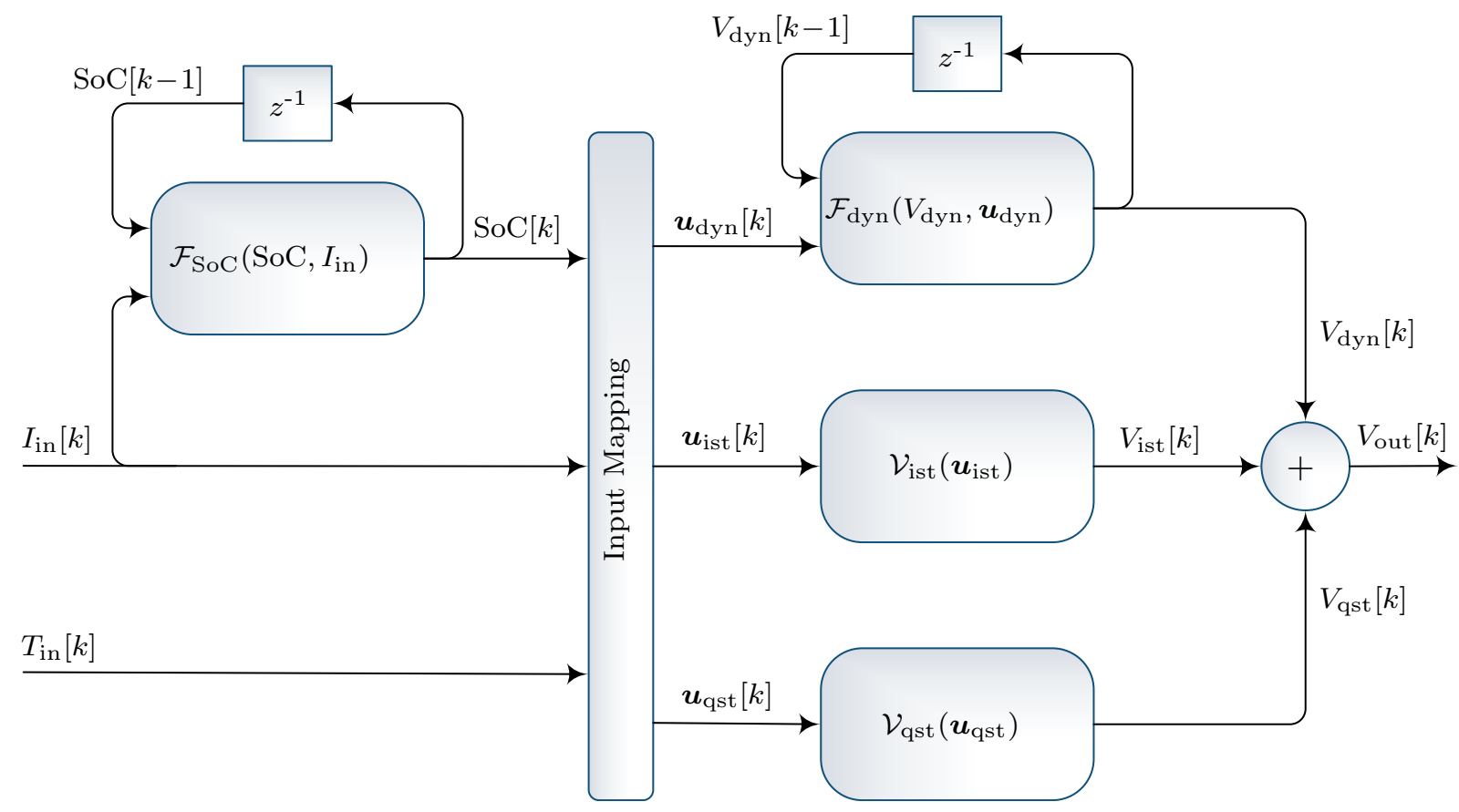

Figure 1. Block diagram of the discrete system for electrochemical cell modeling.

\section{SoC Approximation with Non-Linear Kalman Filter}

As reported in the literature, $\mathrm{KF}$ is an efficient method for SoC estimation $[25,52,53]$. In particular, non-linear versions of KF, such as UKF and EKF have been largely used for this purpose as reviewed in [28], where the authors highlighted the improvements given by non-linear KF with a special emphasis on UKF. 
Non-linear KF is a recursive algorithm that aims at estimating the state vector of a dynamical non-linear discrete system in the presence of additional noise measurement expressed as follows:

$$
\begin{aligned}
\boldsymbol{x}[k+1] & =\mathcal{F}(\boldsymbol{x}[k], \boldsymbol{u}[k])+\boldsymbol{q}[k] \\
\boldsymbol{y}[k] & =\mathcal{G}(\boldsymbol{x}[k], \boldsymbol{u}[k])+\boldsymbol{v}[k]
\end{aligned}
$$

where $x, y$, and $u$ are the state vector, the output vector, and the input vector, respectively. $\mathcal{F}$ and $\mathcal{G}$ are non-linear functions defining the state update and the output equations whilst $\boldsymbol{q}=\mathcal{N}(0, \boldsymbol{Q})$ and $\boldsymbol{v}=\mathcal{N}(0, \boldsymbol{V})$ are the state and output noises drawn from a zero mean gaussian distribution having, respectively, $Q$ and $V$ covariance matrices. The block diagram in Figure 2 shows the main operations performed by the procedure, i.e., prediction and update. The prediction phase is the model-driven part of the algorithm. The available model is exploited for predicting the state evolution and the related output along with their relevant statistics: the state-covariance matrix $\boldsymbol{P}_{x x}$, the output-covariance matrix $\boldsymbol{P}_{y y}$, and the state/output-covariance matrix $\boldsymbol{P}_{x y}$. On the other hand, the update phase exploits a data-driven approach in which the output vector $\boldsymbol{y}_{m}$ measured on the real system is used for updating and correcting the predicted state vector and the state-covariance matrix.

In this work, a non-linear KF has been adopted for the SoC estimation in the electrochemical cell as shown in Figure 3. In particular, the Square Root-Unscented Kalman Filter (SR-UKF) variant [54] has been deployed in order to deal with the non-linearity of the state transition and observation models, as depicted in Equations (1) and (2). Configurations and settings are the same adopted in [46].

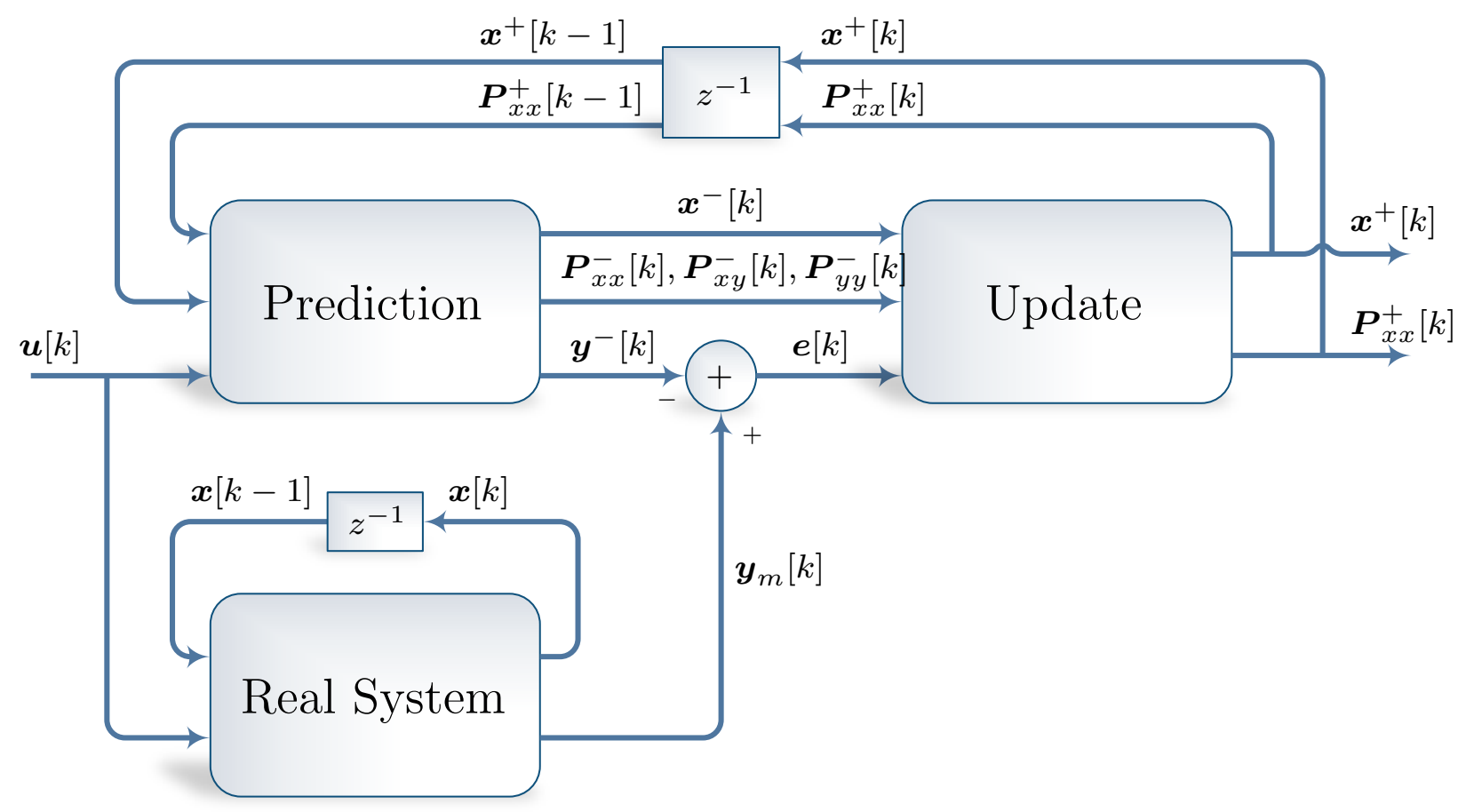

Figure 2. Block diagram of a stochastic state observer. 


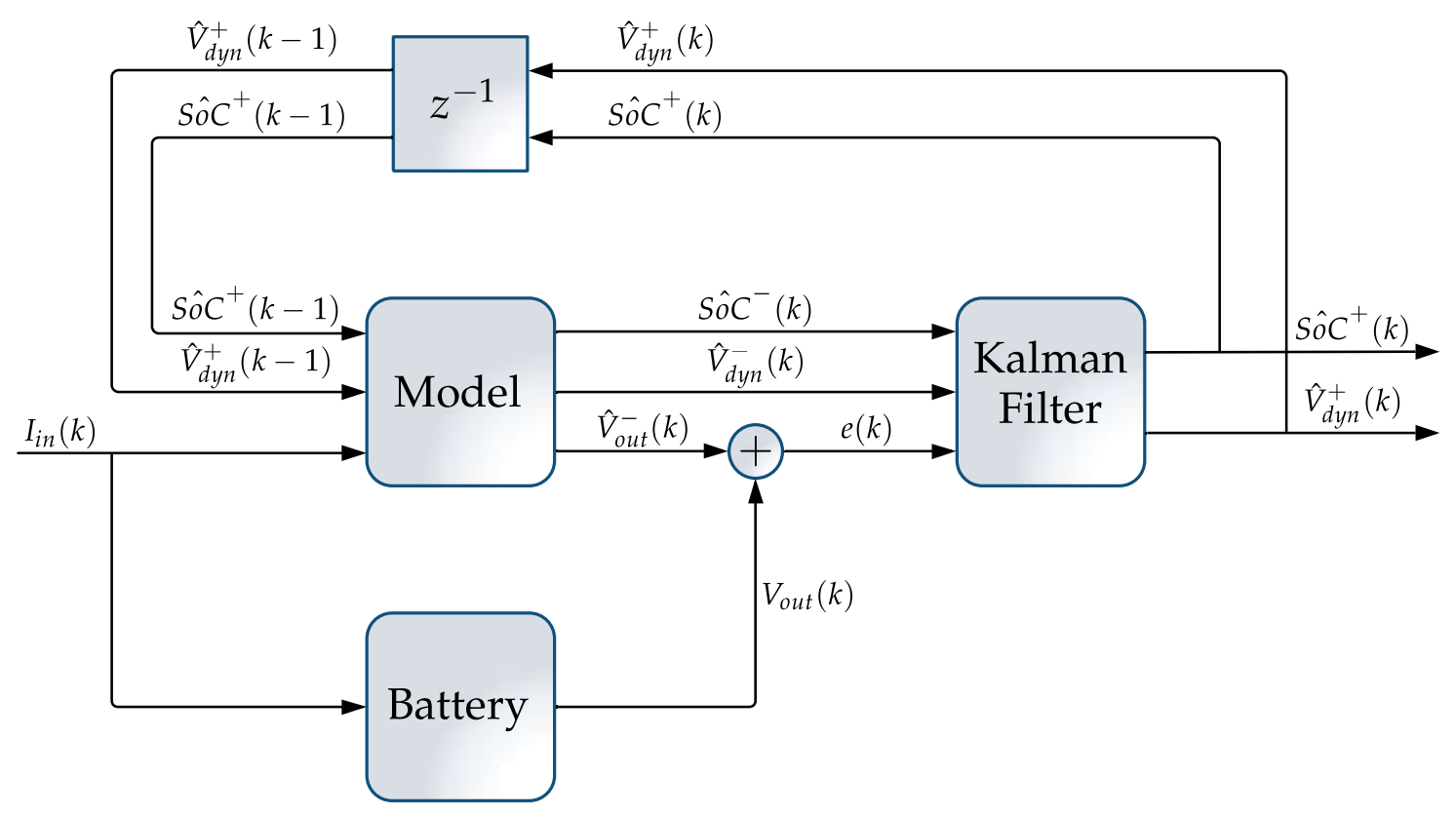

Figure 3. Block diagram of the stochastic non-linear SR-UKF state observer applied to the battery SoC.

\section{Ensemble Neural Network Architecture Adaptation to Equivalent Circuit Model}

The formulation of a suitable circuital model for the battery cell is a critical facet for describing the dynamical behavior of the system. ECM is able to separately estimate the three contributions $V_{q s t}, V_{d y n}$ and $V_{i s t}$ defined in Equations (4)-(6), respectively. This strategy enables the synthesis of a robust and effective prediction model, which keeps the interpretability properties of the ECM. In this section, the ECM is initially described in order to exhaustively explain limitations and advantageous of ENNC architecture.

\subsection{Equivalent Circuit Model}

The purpose of designing an ECM is to adequately reflect the physical behavior of the electrochemical cell. In the ECM, the main voltage quantities (i.e., $V_{q s t}, V_{i s t}$, and $V_{d y n}$ ) are expressed as the electric response of specific circuital elements:

- The instantaneous contribution $V_{\text {ist }}$ is modeled as the voltage drop across the resistor $R_{i s t}$ emulating the internal resistance of the electrochemical cells.

- The dynamic contribution $V_{d y n}$ is schematized as a series of $M$ different $R C$ cells, which aims at modeling the low-pass behavior of the voltage transient response given by the internal charge distribution.

- Being the quasi-stationary contribution $V_{q s t}$ mainly characterized by the amount of charge stored in the cell, it has been modeled as the voltage drop across the capacitor $C_{q s t}$ by means of the OCV-SoC curve. This is the only non-linear component on the whole circuit.

The ECM schematic representing the battery cell is depicted in Figure 4. The ECM parameters identification procedure is based on the analysis of the cell voltage response to a given current profile. For the sake of clarity, Figure 5 can be observed as an example of the voltage contributions, especially the dynamic one, considering a given input current profile constituted by two pulses separated by a resting time interval. 


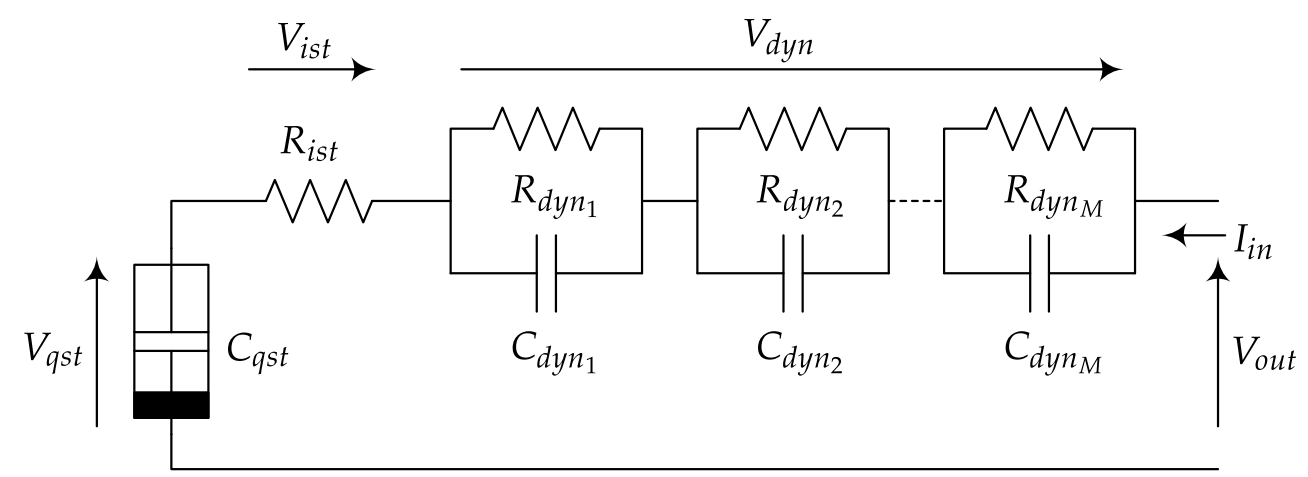

Figure 4. Circuit diagram of the (quasi) linear ECM for a battery cell SoC estimation.
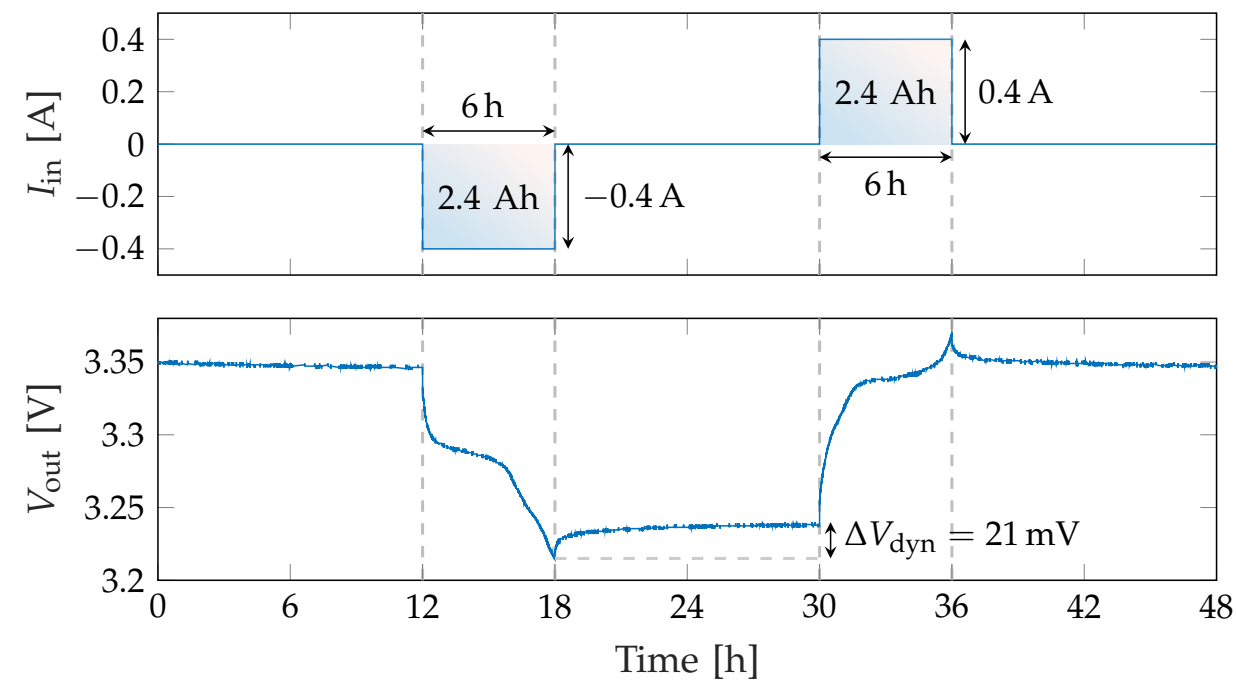

Figure 5. Voltage response of the A123 Nanophosphate AHR23113M1Ultra-B cell to the current profile used for the system identification procedure [40].

Regarding the dynamic contribution, the characteristic equations of a single $R C$ cell are composed by a resistance $R_{d y n}$ and a capacitor $C_{d y n}$, which can be expressed in function of the input current $I_{i n}$ :

$$
I_{i n}(t)=C_{d y n} \frac{d V_{d y n}(t)}{d t}+\frac{V_{C}(t)}{R_{d y n}}
$$

Hence, the dynamic voltage contribution $V_{d y n}$ is evaluated as follows:

$$
V_{d y n}(t)=e^{-\frac{1}{R_{d y n} C_{d y n}}\left(t-t_{0}\right)} V_{d y n}\left(t_{0}\right)+\int_{t_{0}}^{t} e^{-\frac{1}{R_{d y n} C_{d y n}}(t-\tau)} \frac{1}{C_{d y n}} I_{i n}(\tau) d \tau
$$

Moving toward the discrete time domain, Equation (14) is formulated as follows:

$$
V_{d y n}[k+1]=V_{d y n}[k] e^{-\frac{T_{S}}{\tau_{d y n}}}+R_{d y n} I_{i n}[k]\left(1-e^{-\frac{T_{S}}{\tau_{d y n}}}\right)
$$

By considering $M$ different $R C$ cells, the overall dynamic voltage can be expressed as the summation of the individual voltage drops:

$$
V_{d y n}[k]=\sum_{i=1}^{M} V_{d y n, i}[k]
$$

The instantaneous contribution $V_{i s t}$ can be written in the discrete time as the voltage drop across the resistor $R_{i s t}$ : 


$$
V_{\text {ist }}[k]=R_{\text {ist }} I_{\text {ist }}[k]
$$

It is worth noting that in the considered ECM, $\mathcal{R}_{\text {ist }}$ (see Equation (5)) is assumed to be a constant value $R_{i s t}$. Regarding the quasi-stationary contribution, $\mathcal{V}_{q s t}$ takes into account the non-linear behavior of the capacitor $C_{q s t}$, which aims at approximating the cell OCV curve:

$$
V_{q s t}[k]=\mathcal{V}_{q s t}(\operatorname{SoC}[k])
$$

Eventually, the output voltage $V_{\text {out }}$ reads as follows:

$$
V_{\text {out }}[k]=\mathcal{V}_{\text {qst }}(\operatorname{SoC}[k])+\sum_{i=1}^{M} V_{\text {dyn, } i}[k]+R_{\text {ist }} I_{\text {ist }}[k]
$$

The principal drawback of the proposed method regards the $R C$ parameters for defining the transient response of the cell. Indeed, $R_{d y n}$ and $C_{d y n}$ must be correctly defined in order to adequately reflect the transient response, as well as the resistive contribution $R_{\text {ist }}$ given by the instantaneous voltage $V_{i s t}$. Additionally, the number of cell $M$ to employ in order to correctly approximate the low-pass filter behavior is not known a priori. Nonetheless, as the ECM is designed explicitly from a physics hypothesis, it can be interpreted as a white box model with the undeniable advantage of the interpretability aspect. In [40], the authors propose an optimization technique based on swarm intelligence methods (i.e., a PSO algorithm) that automatically select the most suitable parameters of the considered ECM methods. However, the ECM model proposed considers only $I_{i n}$ as input, neglecting other relevant parameters as the operating temperature and the $S o C$ influences on $V_{d y n}$ and $V_{i s t}$. Indeed, $R_{i s t}, R_{d y n, i}$ and $C_{d y n, i}$ are constant values that do not change once optimized.

\subsection{ENNC Model}

The limitation imposed by the ECM model has been faced with introducing an ensemble of NNs [50], which individually model the non-linear behavior of the circuital components described in Section 4.1. Specifically, the ENNC approach [46] is able to preserve the interpretability aspect characterizing the ECM and simultaneously solving the main issues regarding the inability to take into account the relevant input parameters. In the block diagram shown in Figure 6, it is possible to spot how the circuital component has been displaced in favor of specific NNs while maintaining the same ECM circuital architecture. Even though the resistors components $R_{i s t}, R_{d y n}$ still respect the constitutive electrical law (i.e., $V=R I$ ) their values are instead non-linear functions of $I_{i n}, T_{i n}$, and SoC. The same discussion holds for $C_{q s t}$ and $C_{d y n}$ : the capacitor still impose a voltage drop defined by the equation $V=\frac{1}{C} \int I d t$ but their capacitance values are individually defined as non-linear functions of the relevant parameters according to the NNs outputs. The system equations read as follows:

$$
\begin{gathered}
{\left[\begin{array}{c}
S o C[k+1] \\
V_{d y n_{1}}[k+1] \\
\vdots \\
V_{d y n_{N}}[k+1]
\end{array}\right]=\mathcal{A}\left(\mathbf{u}_{\mathbf{d y n}}[k]\right)\left[\begin{array}{c}
S o C[k] \\
V_{d y n_{1}}[k] \\
\vdots \\
V_{d y n_{N}}[k]
\end{array}\right]+\mathcal{B}\left(\mathbf{u}_{\mathbf{d y n}}[k]\right) I_{i n}[k]} \\
V_{\text {out }}[k]=\mathcal{V}_{q s t}\left(\mathbf{u}_{\mathbf{q s t}}[k]\right)+\sum_{i=1}^{M} \omega_{d y n_{i}} V_{d y n, i}[k]+\mathcal{R}_{\text {ist }}\left(\mathbf{u}_{\text {ist }}[k]\right) I_{\text {ist }}[k]
\end{gathered}
$$

In Equation (21), $M$ is the state space dimension where $M=N+1$, with $N$ being the number of $R C$ bipoles that model the dynamic behavior of the cell. Furthermore, $\mathcal{V}_{q s t}$ is the OCV-SoC curve that gives the indication about $C_{q s t}, \omega_{d y n}$ and $V_{d y n}$ are respectively the $R C$ weights and the output values, whilst $\mathcal{R}_{\text {ist }}$ is the non-linear function modeling the instantaneous response of the cell with the responsive behavior. 


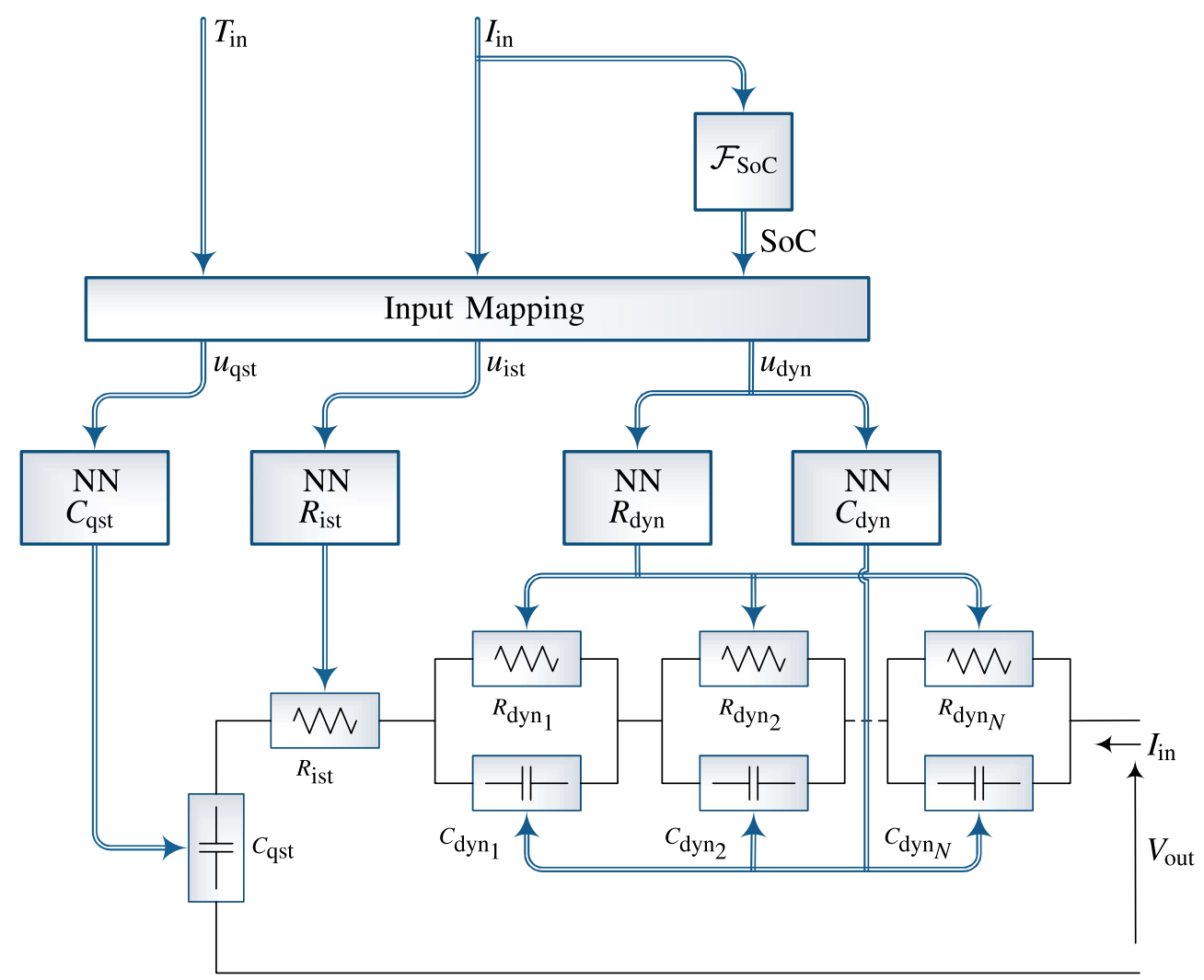

Figure 6. ENNC model adaptation to the ECM.

The state equations shown in Equation (20) are described according to $\mathcal{A} \in \mathbb{R}^{M \times M}$ and the vector $\mathcal{B} \in \mathbb{R}^{M \times 1}$ :

$$
\begin{aligned}
& \mathcal{A}\left(\mathbf{u}_{\mathbf{d y n}}[k]\right)=\left[\begin{array}{cccc}
1 & 0 & \ldots & 0 \\
0 & e^{\frac{-T_{S}}{\bar{d}_{\text {dyn }}\left(\mathbf{u}_{\mathbf{d y n}}[k]\right)}} & \ldots & 0 \\
\vdots & \ldots & \ddots & \vdots \\
0 & 0 & \ldots & e^{\frac{-T_{S}}{\tau_{d y n}\left(\mathbf{u}_{\mathbf{d y n}}[k]\right)}}
\end{array}\right] \\
& \mathcal{B}\left(\mathbf{u}_{\mathbf{d y n}}[k]\right)=\left[\begin{array}{c}
\frac{T_{S}}{C_{n}} \\
\left(1-e^{\frac{-T_{S}}{\left.\bar{d}_{\text {dyn }} \mathbf{u}_{\mathbf{d y n}}[k]\right)}}\right) \mathcal{R}_{d y n_{1}} \mathbf{u}_{\mathbf{d y n}}[k] \\
\vdots \\
\left(1-e^{\left.\frac{-T_{S}}{\bar{\tau}_{d y n_{N}\left(\mathbf{u}_{\mathbf{d y n}}[k]\right)}}\right)} \mathcal{R}_{d y n_{N}} \mathbf{u}_{\mathbf{d y n}}[k]\right.
\end{array}\right]
\end{aligned}
$$

The non-linear functions $\mathcal{V}_{q s t}, \mathcal{R}_{i s t}, \mathcal{R}_{d y n}$ and $\mathcal{T}_{\text {dyn }}$ are individually defined by four different NNs, which shall reflect their basic properties:

- $\quad \mathcal{R}_{\text {ist }}$ is a Multi Layer Perceptron (MLP) NN that evaluates $R_{i s t}$ in Equation (17) starting from the input vector $\mathbf{u}_{i s t}$ (see Equation (10)). Hence, its output layer is limited to a single neuron.

- $\quad \mathcal{R}_{d y n}$ and $\mathcal{T}_{d y n}$ are both MLP networks taking in input $\mathbf{u}_{d y n}$ as defined in Equation (9). In their output layers, the networks are equipped with exactly $N$ neurons according to the number of $R C$ cells employed for the dynamic contribution. Hence, their output values are respectively $R_{d y n}=\left\{R_{d y n_{1}}, \ldots, R_{d y n_{N}}\right\}$, and $\tau_{d y n}=\left\{\tau_{d y n_{1}}, \ldots \tau_{d y n_{N}}\right\}$.

- $\quad \mathcal{V}_{q s t}$ is addressed by a Functional Link Neural Network (FLNN) since it has been already shown effective capabilities in the approximation of the OCV-SoC curve [46]. 
The input vector $\mathbf{u}_{q s t}$ takes into account only the temperature $T_{\text {in }}$ and $S o C$ values. Indeed, the currents used for the OCV curve generation are negligible. Moreover, this model constraint is necessary for avoiding any kind of conflict between the MLP modeling $\mathcal{R}_{\text {ist }}$ and the FLNN modeling $V_{\text {qst }}$.

The ENNC architecture implementing the NNs blocks is shown in Figure 7.

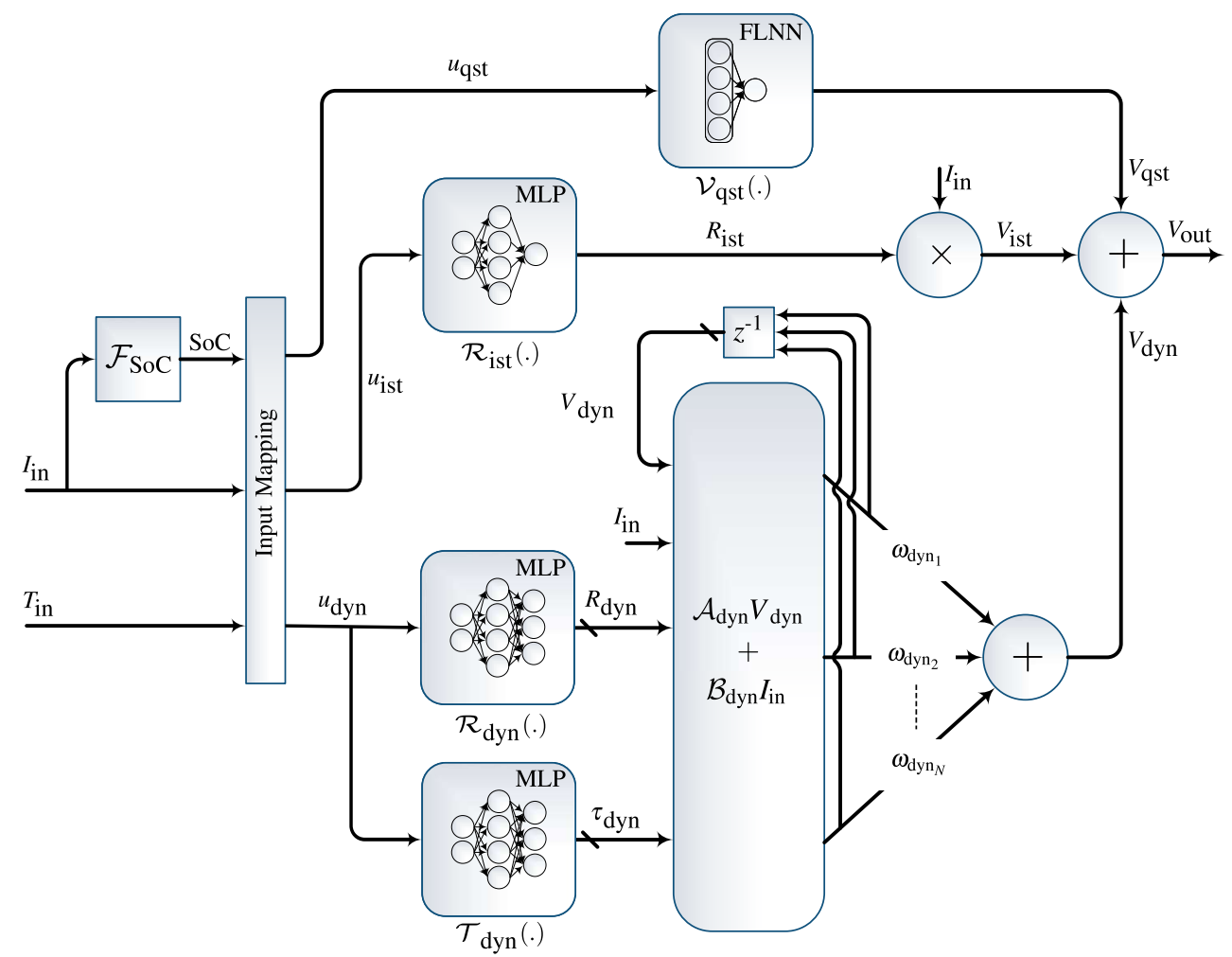

Figure 7. ENNC block diagram.

In addition to the three MLP NNs and the FLNN, a single-layer Recurrent Neural Network (RNN) is placed after the MLP NNs indicated as $\mathcal{R}_{d y n}$ and $\mathcal{T}_{d y n}$. The RNN applies the state equation (Equation (20)) reading in input the cell current $I_{i n}$, the dynamic voltage contribution evaluation at the previous step and the output generated by the NNs $\mathcal{T}_{d y n}$ and $\mathcal{R}_{d y n}$, namely the vectors $R_{d y n}$ and $\tau_{d y n}$. The RNN has an output layer defined by the RC weights $\omega_{d y n}$ (see (21)). The $\omega_{d y n}$ training allows to better tune the RC voltage contribution, especially in case more RC filters are considered.

The application of NN methods for modeling electrochemical cells has been previously explored in many different studies that involved a plethora of architectures [28,43-45,55-57]. Nonetheless, these approaches do not take into account any assumption about the physical properties regarding the battery cell. Indeed, the NNs approaches employed are classified as black-boxes [53], since these architectures only provide the prediction of the system response without giving any insight regarding the underlying process. Even though this approach can provide accurate predictions, the lack of interpretability poses several issues for understanding the critical state of the cell.

The ENNC approach discussed here can be considered instead as a white-box model since the electrical behavior of the whole system is described by the ECM whose assumptions have already been physically motivated. As a universal functional approximator, $\mathrm{NN}$ gives the chance to model accurately the non-linear components that characterize the process and additionally keeps the interpretability aspect offered by the deployment of the ECM model unaltered. 


\section{Physical Inspired Equivalent Neural Network Circuit Model}

In this section, the ENNC architecture has been improved by taking into account the electrochemical model based on the fractional order ESP formulation proposed in [34] in order to design a physical inspired model called PI-ENNC.

The study of Fan G. et al. [34] is mainly focused on evaluating the effectiveness of the transfer functions formulation on the Laplacian domain in order to accurately approximate all the cell physics defined by the Extended Single Particle (ESP) model equations and constraints. The ESP model is a physical-inspired mathematical model [58-60] in which the cell is simplified by two main (solid) spherical particles immersed in a (liquid) electrolyte where the chemical properties and the cell geometry are properly defined by a given set of parameters. The ESP modeling follows the main assumption that has already been used in the conventional single particle models [61] considering a uniform electrochemical reaction along the $\mathrm{x}$-dimension in each electrode. However, in order to improve the accuracy and expand the applicability of the model, the ohmic effects and charge distribution in the electrolyte phase are also taken into consideration.

In ESP modeling, the voltage contributions are defined by the partial difference equations, which describe the physical dynamics of the cell. Specifically, there is no direct relationship between the potentials, the voltage transients and the applied current. These aspects make it difficult to establish the state transition equations, analyze observability and apply methods for estimating the state of the system.

It is clear that all these aspects impose relevant limitations for the battery SoC prediction in automotive applications. Hence, the equations of the ESP model have been simplified by Fan G. et al. in [34] through a fractional order approximation on the solidphase diffusion contribution after formulating the relationship between the load current and the surface concentration of the solid particle according to a proper transfer function. Finally, all the simplifications lead to the formulation of a first-order transfer function, which further reduces the computational costs of the SoC estimation.

In the following section (Section 5.1), the transfer functions adopted in [34] are commented and compared with the formulation introduced in Section 2. Indeed, although the work carried out by Fan G. et al. has a completely different approach centered on the transfer functions formulation and the tuning of the physical parameters without the use of machine learning and data driven techniques, it is possible to observe a strong analogy with the problem formulation of Section 2 by analysing the voltage contributions and their relationship with the load current.

\subsection{Analogy between the Physics-Based Fractional Order Model and the ENNC Model}

In ESP models, the voltage contributions (overpotentials in [34]) have been summarized in four different terms:

- The ohmic effect $\eta_{\text {ohm }}$;

- The electrolyte diffusion $\eta_{e, \text { con }}$;

- The solid state diffusion of both anode and cathode particles $U_{s, a}^{\text {surf }}$ and $U_{s, c}^{\text {surf }}$, respectively.

The terminal voltage $V_{\text {cell }}(s)$ is finally expressed as follows:

$$
V_{\text {cell }}(s)=U_{s, p}^{\text {surf }}(s)+U_{s, n}^{\text {surf }}(s)+\eta_{e, \text { con }}(s)+\eta_{\text {ohm }}(s)
$$

The transfer functions of each overpotential mentioned in [34] are described below focusing on the relationship with the load current $I_{L}$ in order to apply an analogy with the voltage contributions discussed in Section 2:

1. Ohmic effect and electrochemical reaction description: these two contributions are lumped together since they can be approximated as a zero-order transfer function with respect to $I_{L}$ :

$$
\frac{\eta_{o h m}(s)}{I_{L}(s)}=-\theta_{R_{0}}
$$


The parameter $\theta_{R_{0}}$ summarizes all the ohmic effects such as the current collector, the electrolyte and the film resistances and the electrochemical reaction overpotential. It has the same properties of the instantaneous contribution, therefore $\theta_{R_{0}}$ can be referred to as $\mathcal{R}_{\text {ist }}$ of Equation (5).

2. Electrolyte diffusion approximation: the relationship between the electrolyte concentration overpotential (i.e., voltage drop) and the load current can be presented by a first-order RC equivalent circuit:

$$
\frac{\eta_{e, \text { con }}(s)}{I_{L}(s)}=-\frac{\theta_{R e}}{\theta_{R e} \theta_{C e} s+1}
$$

where the parameters $\theta_{R e}$ and $\theta_{C e}$ summarize the electrolyte concentration dynamics and the cell geometry after applying an important simplification on the model with a first-order Taylor expansion method. It is analogous to the dynamic contribution $V_{d y n}$ in Equation (4).

3. Solid state diffusion overpotential: it is applied on both the electrodes (positive- $\mathrm{p}$ and negative $-n$ ) and it is a function of the particle surface concentration $C^{\text {surf }}$ formulated as follows:

$$
U_{s, i}^{\text {surf }}=f_{E_{o c, i}}\left(C_{s, i}^{\text {surf }} / C_{s, i}^{\max }\right) i \in\{p, n\}
$$

where, $f_{E_{o c, i}}$ is a non-linear function analogously to the quasi-stationary contribution $V_{q s t}$. However, the surface concentration $C^{\text {surf }}$ is not only a function of the active particle volume average charge concentration called $C^{\text {avg }}$ but also depends on a dynamic component called $C^{\operatorname{diff}}$, namely:

$$
C_{s, i}^{s u r f}=C_{s, i}^{a v g}+C_{s, i}^{\operatorname{diff}}
$$

$C_{s, i}^{\text {diff }}$ represents the concentration difference between the surface and the volume average value of the active particle which is function of the ion pore wall flux of the cell, and therefore of the density current. Finally, the transfer function related to $C_{s, i}^{\text {surf }}$ has been found and tested after applying a proper simplification and truncation to the first-order to reduce the computational cost (see Equation (21) in [34]):

$$
\frac{C_{s, i}^{\text {surf }}(s)}{I_{L}(s)}=( \pm) \underbrace{\frac{1}{3 \theta_{Q_{i} s}}}_{\substack{C_{s, i}^{\text {ave }} \\ I_{L}(s)}}+\underbrace{\frac{0.2+0.0461 \theta_{D_{S, i}}^{-1} s^{0.5}}{1+0.2066 \theta_{D_{S, i}}^{-1} s^{0.5}+0.0510 \theta_{D_{S, i} s}^{-2} s}}_{\frac{C_{S, i}^{\text {diff }}}{I_{L}(s)}}
$$

where the parameters $\theta_{D_{S, i}}^{-1}$ and $\theta_{Q_{i}}$ represent the particle properties and their interactions with the electrolyte. These are functions of the solid diffusion coefficient, the electrode surface area, the volume fraction of the active material, the particle radius, the activity coefficient and other geometry properties of the cell.

Taking inspiration from the study in [34], in the model proposed in this work the differential solid state diffusion overpotential in (29) has been re-interpreted considering also the differential contribution, which we call dynamic. Specifically, in this work, the dynamic contribution on the SoC is approximated through a first-order RC filter in the ENNC. It is worth noting that with the latter assumption, the ENNC should also consider the input current in the input array $\boldsymbol{u}_{\text {qst }}$ conversely to the discussion held in Section 4.2.

\subsection{PI-ENNC Architecture}

As formulated in [34], the overall particles charge at the surface is the sum of the average volume $C^{a v g}$ charge and a differential term $C^{\text {diff }}$ (see Equation (28)). In this work, 
a dynamic term of the SoC named $S_{0} C_{d y n}$ is introduced, holding the same properties of the differential term $C^{\text {diff }}$. Therefore, the SoC can be written as:

$$
S o C_{t o t}=S o C_{a v g}+S o C_{d y n}
$$

where the term $S o C_{a v g}$, which can be associated with $C^{a v g}$ in Equation (28), corresponds to the SoC calculated through the CC algorithm in Equation (8).

According to [34], the dependency of the $S o C_{d y n}$ with respect to $I_{i n}$ can be approximated by a first-order low pass filter as in Equation (29). Therefore, the discreet form of $S o C_{d y n}$ can be calculated as in Equation (23), namely:

$$
S o C_{d y n}[k+1]=S o C_{d y n}[k] e^{-\frac{T_{s}}{\tau_{q s t}}}+R_{q s t} I_{i n}[k]\left(1-e^{-\frac{T_{s}}{\tau_{q s t}}}\right)
$$

However, in this case, $\tau_{q s t}$ and $\mathcal{R}_{q s t}$ do not represent a resistance and a time constant. These symbols have been used just for respecting the analogy with the low pass behavior of an RC circuit.

Concerning PI-ENNC model architecture, the $S o C_{d y n}$ term is estimated with a similar NN configuration adopted for $V_{d y n}$. As shown in Figure 8, two MLP NNs $\mathcal{R}_{q s t}$ and $\mathcal{T}_{q s t}$ are in charge of calculating $R_{q s t}$ and $\tau_{q s t}$, respectively. In this case, the MLP input $\mathbf{u}_{q s t d y n}$ is composed of the only current $I_{i n}$ in order to respect the formulation in Equation (29). Finally, no changes have been applied for the dynamic and instantaneous voltage contribution with respect to the ENNC model.

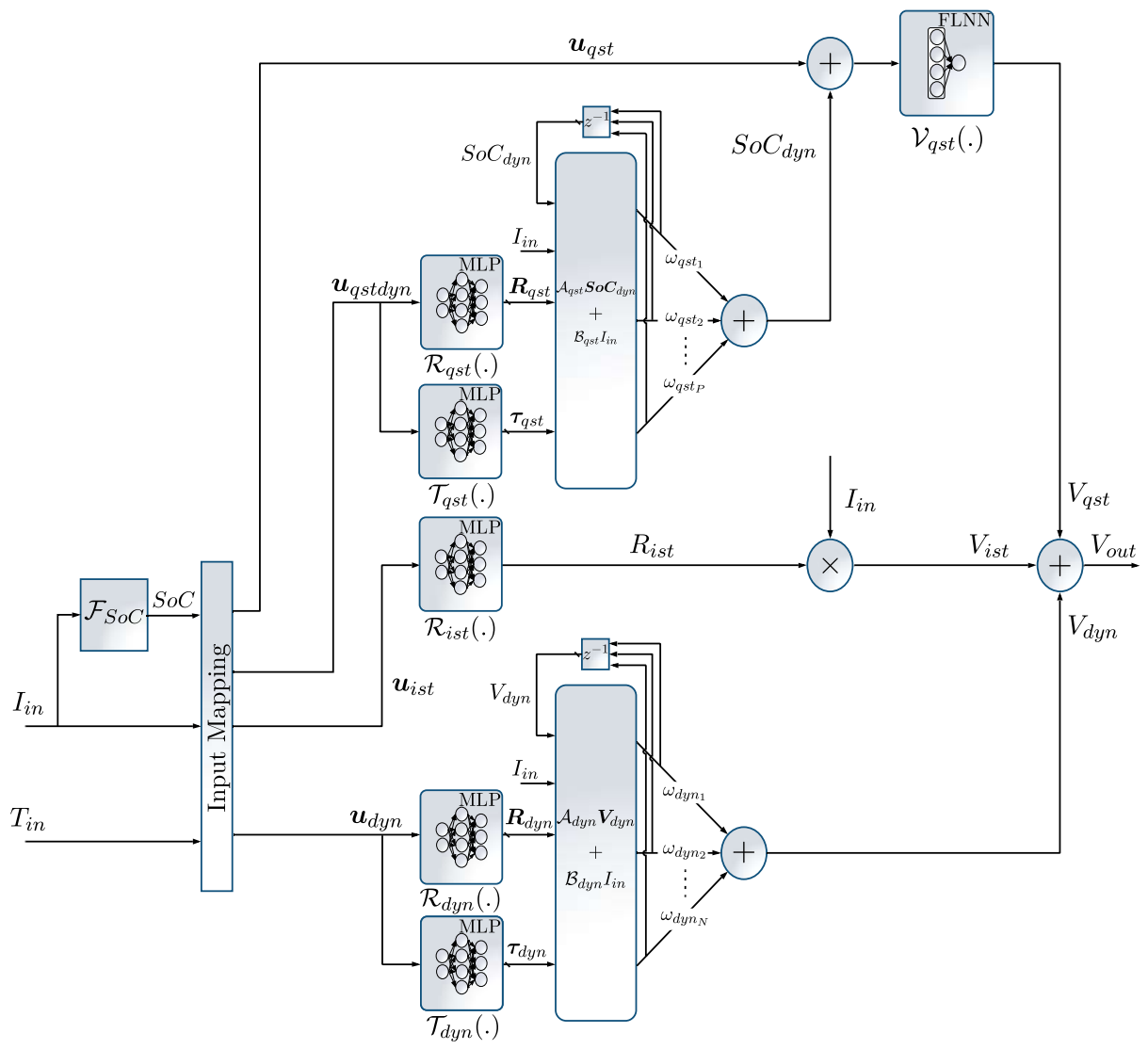

Figure 8. PI-ENNC block diagram.

\section{Dataset}

The NN model blocks were trained and tested according to three different datasets that have been extensively employed in previous works [62]. 


\subsection{Randomized Battery Usage Data Set}

The first dataset is the Randomized Battery Usage Data Set collected by NASA Ames Research Center [63]. It is a collection of measurements performed on a 2.1 Ah Lithium-Ion cell model 18650. The cell was tested with randomly generated sequences of charge and discharge pulses. Starting from a fully charged cell in a stationary condition (i.e., with SoC $=100 \%$ and $V_{d y n}=0$ ), each pulse of the sequence is randomly selected from the set:

$\{-4.5 A,-3.75 A,-3 A,-2.25 A,-1.5 A,-0.75 A, 0.75 A, 1.5 A, 2.25 A, 3 A, 3.75 A, 4.5 A\}$

Consequently, the sampled pulse is applied to the cell until the output is in the range $[3.2 \mathrm{~V}, 4.2 \mathrm{~V}]$ or according to a maximum time frame.

The applied current $I_{i n}$, the output voltage $V_{\text {out }}$ and the surface temperature $T_{\text {in }}$ have been measured with a sampling time of $1 \mathrm{~s}$. The $\mathrm{SoC}$ sequence is obtained by using the CC algorithm (see Equation (8)). The training set includes the first 100 pulses of the first random cycle test performed on the cell ID RW9. Similarly, the test set is composed of the first 100 pulses related to the test of the second random cycle of the same cell. The time series $I_{\text {in }}, V_{\text {out }}, T_{\text {in }}$ and $S o C$ are shown in Figure 9, whereas in Figure 10, it is in shown the cell OCV curve.
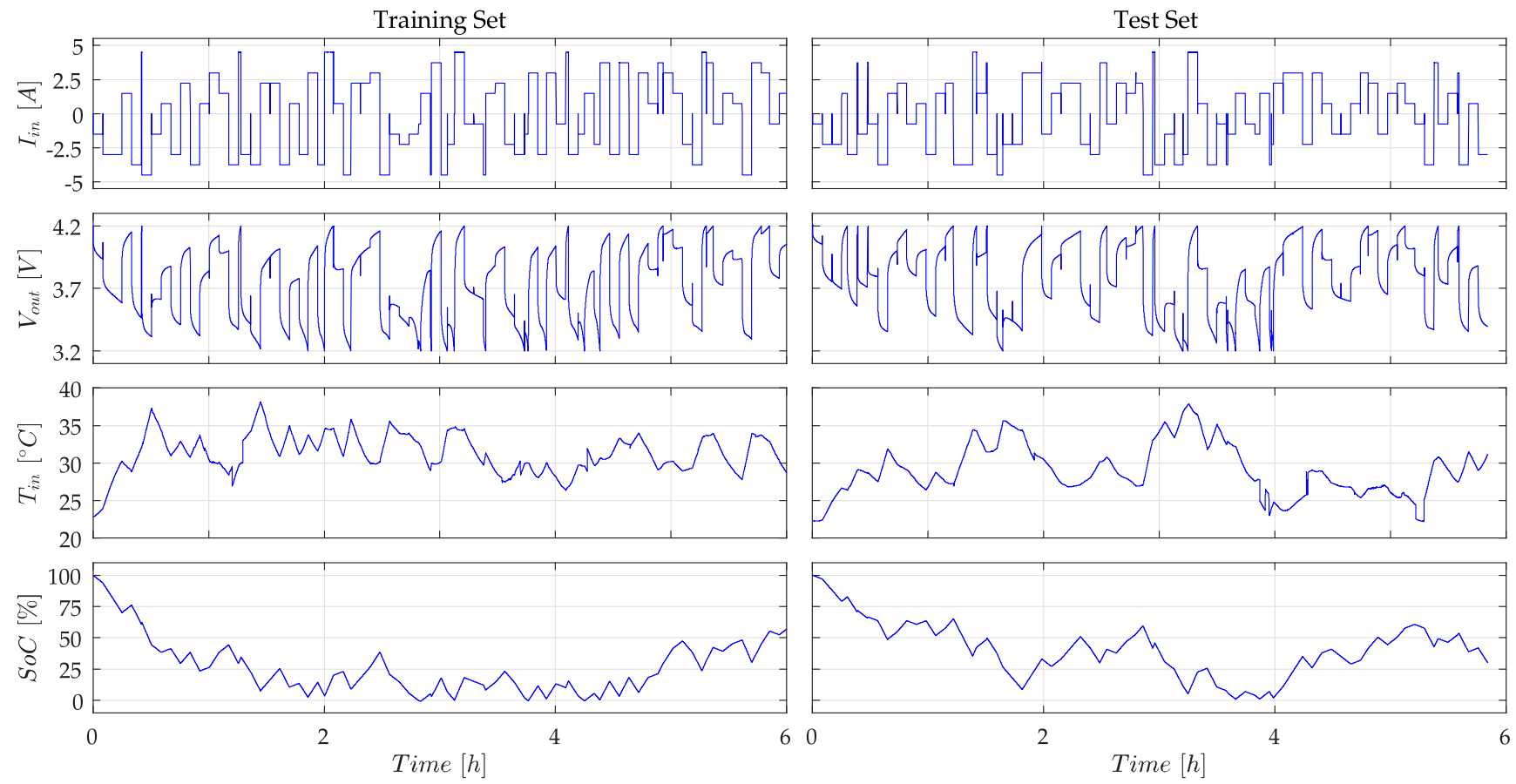

Figure 9. $I_{\text {in }}, V_{\text {out }}, T_{\text {in }}$, and $S o C$ time series referring to the Randomized Battery Usage-NASA dataset.

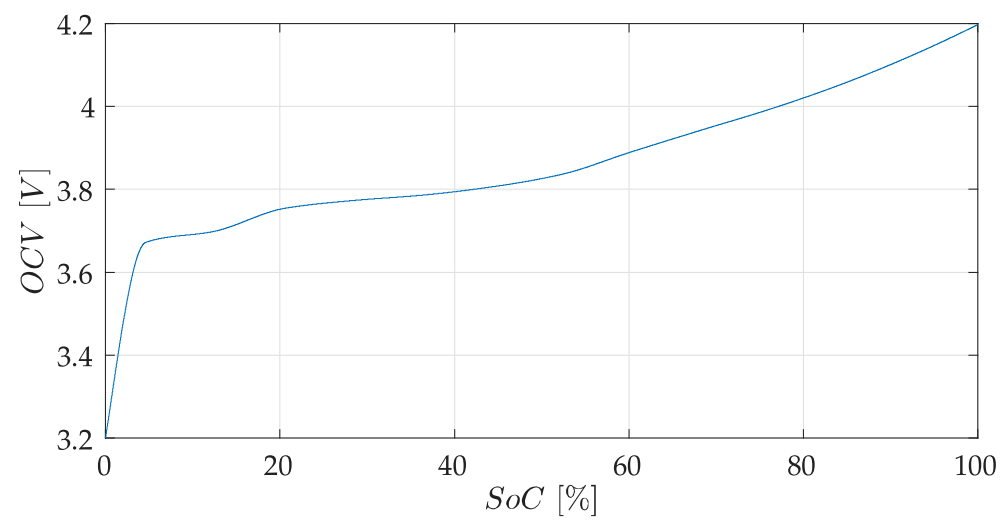

Figure 10. OCV-SoC curve referring to the NASA battery cell. 


\subsection{Dataset A123 Cell}

The second dataset considered refers to A123 Lithium-Ion cell [64]. The cell parameters are shown in Table 1.

Table 1. A123 Cell Parameters.

\begin{tabular}{cccccc}
\hline Type & $\begin{array}{c}\text { Nominal } \\
\text { Voltage }\end{array}$ & $\begin{array}{c}\text { Nominal } \\
\text { Capacity }\end{array}$ & $\begin{array}{c}\text { Upper/Lower } \\
\text { Cut-Off Voltage }\end{array}$ & $\begin{array}{c}\text { Maximum Continuous } \\
\text { Discharging Current }\end{array}$ & $\begin{array}{c}\text { Usage } \\
\text { Temperature }\end{array}$ \\
\hline $\mathrm{LiFePO} 4$ & $3.3 \mathrm{~V}$ & $1.1 \mathrm{Ah}$ & $3.6 \mathrm{~V} / 2.0 \mathrm{~V}$ & $30 \mathrm{~A}\left(\right.$ at $\left.25{ }^{\circ} \mathrm{C}\right)$ & $-30{ }^{\circ} \mathrm{C}$ to $+50{ }^{\circ} \mathrm{C}$ \\
\hline
\end{tabular}

The dataset is composed of three different usage profiles:

- $\quad$ Dynamic Stress Test (DST).

- Federal Urban Driving Schedule (FUDS).

- US06, Highway Driving Schedule. (HDS)

The cycle was designed by the United States Advanced Battery Consortium and simulates a dynamic discharge regime whose full cycle is 360s. The DST has been used as a training set as suggested in [65], whereas the FUDS and US06 as test sets since they are characterized by more sophisticated dynamic profiles. In fact, these tests are more complex than DST in terms of charge and discharge current rates. The current time series are shown in Figure 11. The measurements are sampled every 1s. The SoC is estimated according to the CC algorithm in Equation (8). The DST, FUDS and US06 datasets time series are shown in Figure 12, whilst the OCV curve is shown in Figure 13.
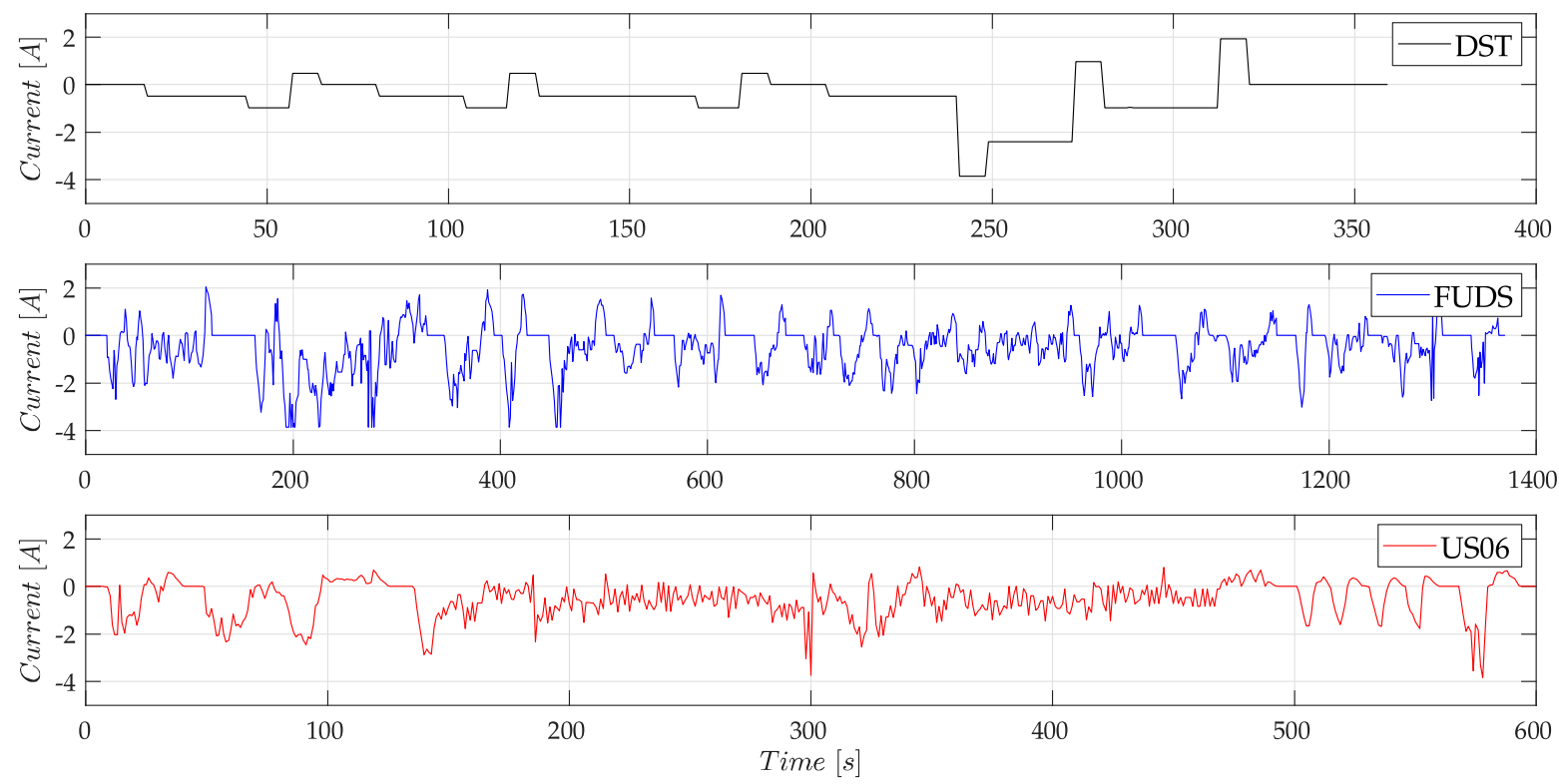

Figure 11. Dynamic test profile showing the applied current for A123 dataset: Dynamic Stress Test (DST), Federal Urban Driving Schedule (FUDS) and US06 Highway Driving Schedule (US06). 

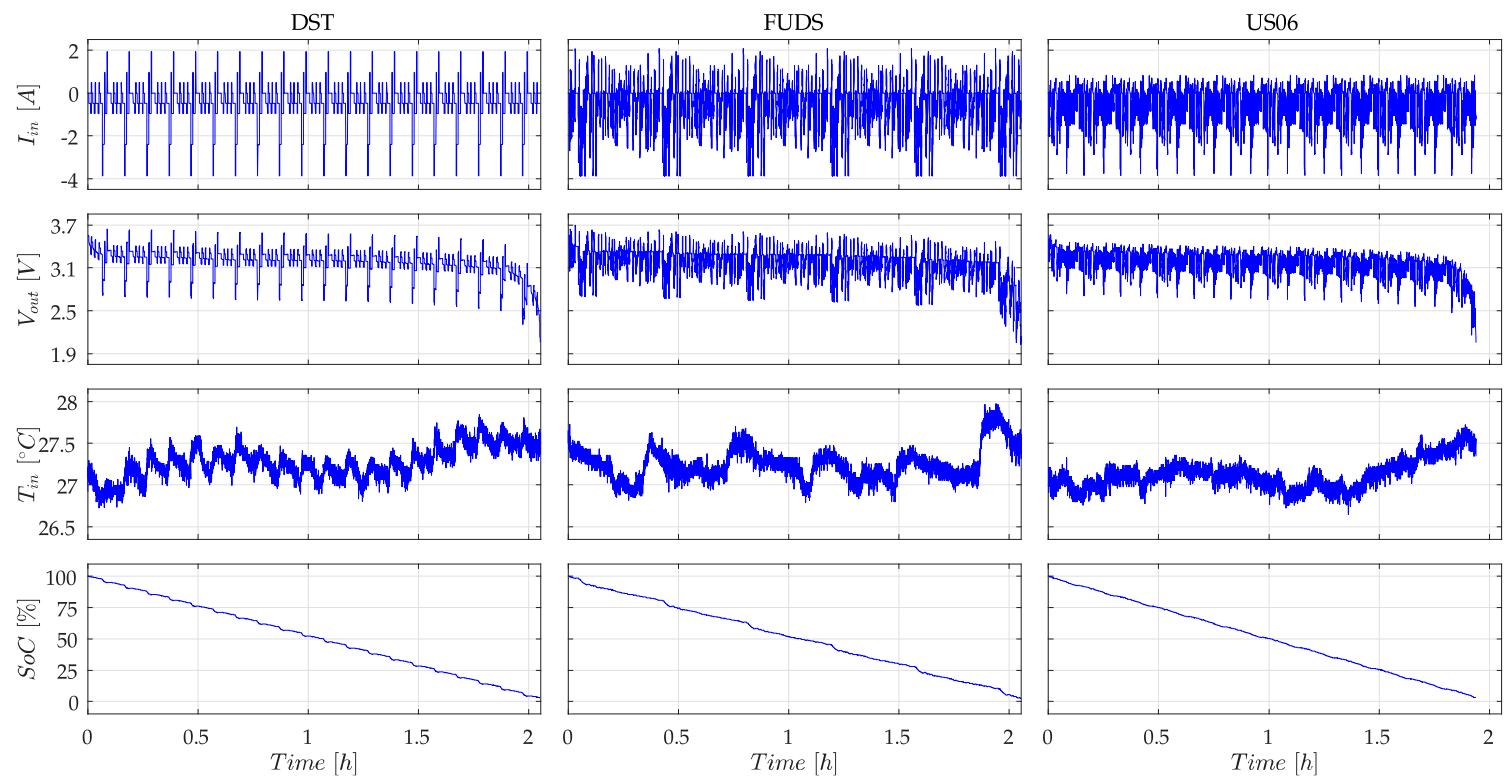

Figure 12. A123 cell time series $I_{\text {in }}, V_{\text {out }}, T_{i n}, S o C$ of the dataset DST, FUDS and US06.

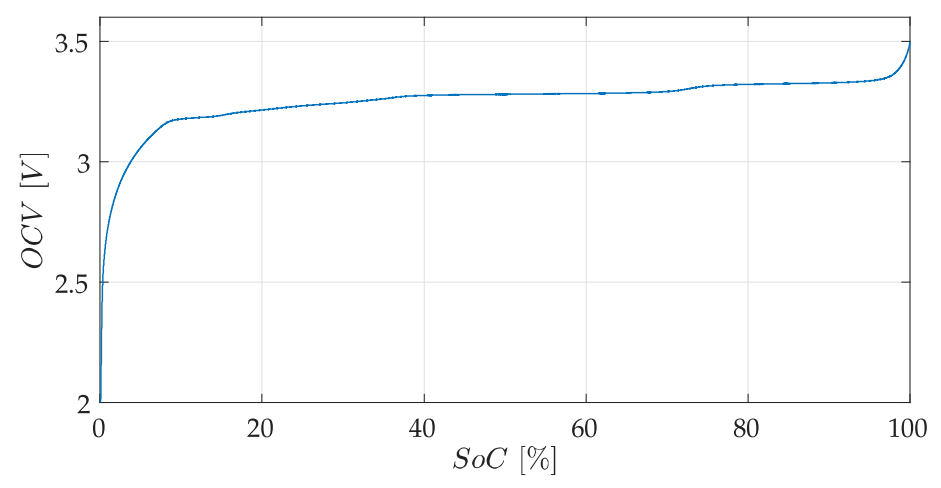

Figure 13. OCV-SoC curve referred to the A123 battery cell.

\subsection{Dataset INR 18650-20R}

The last dataset is named INR 18650-20R Lithium-ion cell [64] whose parameters are shown in Table 2. The datasets provide four different Dynamic Test Profiles (DTPs):

- $\quad$ Dynamic Stress Test (DST).

- Federal Urban Driving Schedule (FUDS).

- US06 Highway Driving Schedule.

- $\quad$ Beijing Dynamic Stress Test (BJDST).

As in dataset A123, the DST has been used as a training set whilst the remaining profiles are employed as test sets for assessing the BMS model performance. The overall cycles times of FUDS, US06 and BJDST are, respectively, 1372s, 600s and 916s. The respective current time series are shown in Figure 14. The sampling time is fixed at 1s and the SoC is measured according to Equation (8). The DST, FUDS, US06 and BJDST time series are illustrated in Figure 15, whereas the SoC-OCV curve is shown in Figure 16.

Table 2. INR 18650-20R cell parameters.

\begin{tabular}{cccccc}
\hline Type & $\begin{array}{c}\text { Nominal } \\
\text { Voltage }\end{array}$ & $\begin{array}{c}\text { Nominal } \\
\text { Capacity }\end{array}$ & $\begin{array}{c}\text { Upper/Lower } \\
\text { Cut-Off Voltage }\end{array}$ & $\begin{array}{c}\text { Maximum Continuous } \\
\text { Discharging Current }\end{array}$ & $\begin{array}{c}\text { Usage } \\
\text { Temperature }\end{array}$ \\
\hline $18650 \mathrm{LiNiMnCoO} 2 /$ Graphite & $3.6 \mathrm{~V}$ & $2.0 \mathrm{Ah}$ & $4.2 \mathrm{~V} / 2.5 \mathrm{~V}$ & $22 \mathrm{~A}\left(\right.$ at $\left.25^{\circ} \mathrm{C}\right)$ & $0{ }^{\circ} \mathrm{C}$ to $+50^{\circ} \mathrm{C}$ \\
\hline
\end{tabular}


(a)

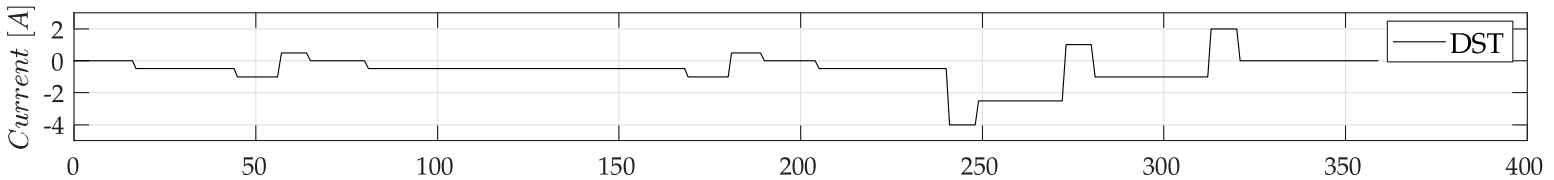

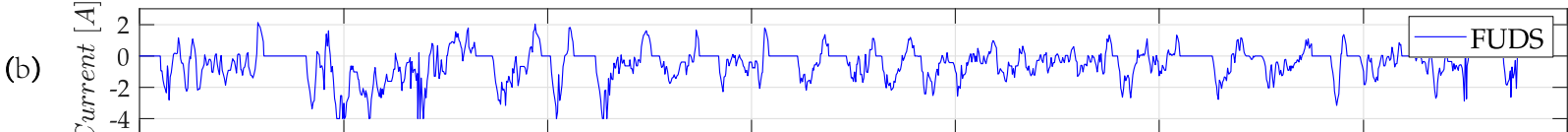

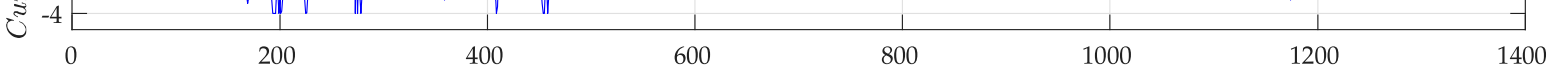

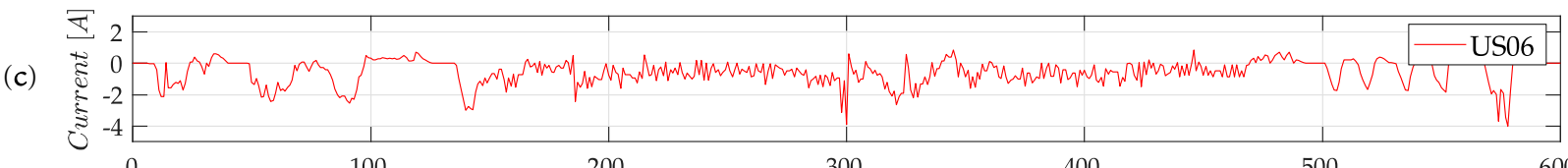

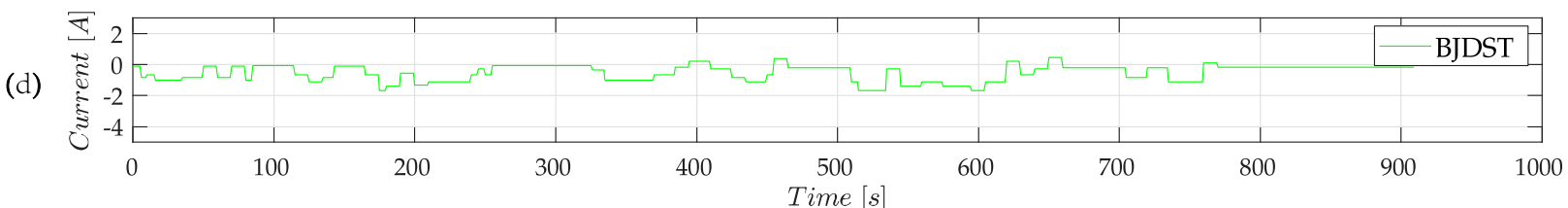

Figure 14. Dynamic test profile showing the applied current for the INR 18650-20R dataset: (a) Dynamic Stress Test, (b) Federal Urban Driving Schedule, (c) US06 Highway Driving Schedule e (d) Beijing Dynamic Stress Test.
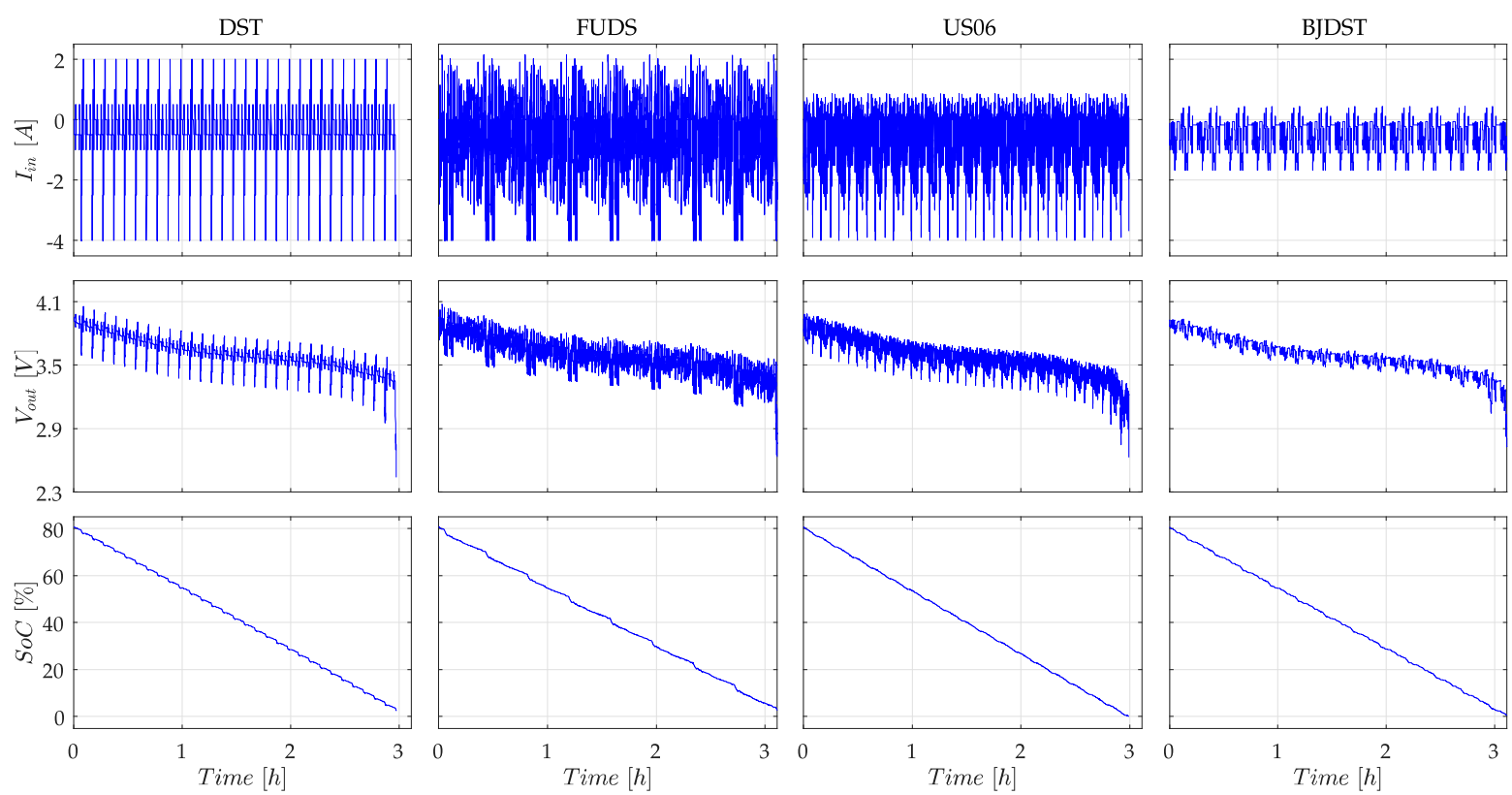

Figure 15. INR 18650-20R cell time series $I_{\text {in }}, V_{\text {out }}, T_{\text {in }}$, SoC of the dataset DST, FUDS and US06.

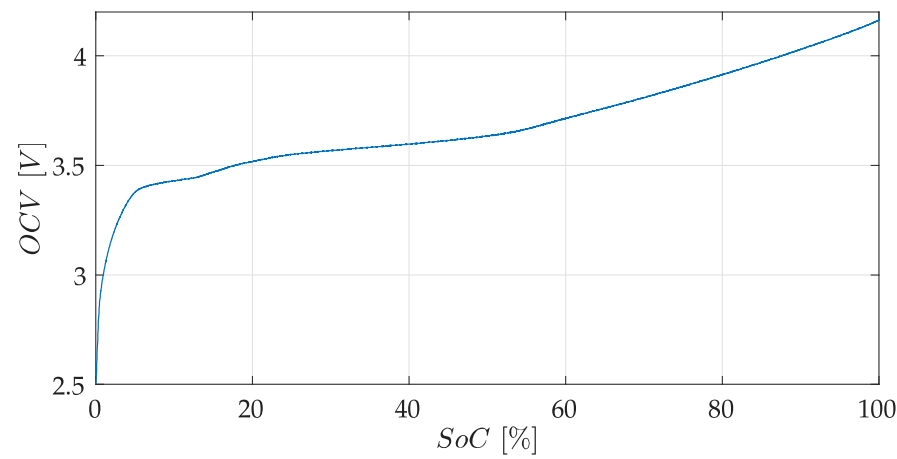

Figure 16. OCV-SoC curve referred to the INR 18650-20R battery cell. 


\section{Test Settings}

The PI-ENNC model discussed in Section 5 is compared with the ENNC considering the SoC approximation as the performance index according to the BMS system introduced in Section 2. The NNs composing both PI-ENNC and ENNC have been developed in Python with the Keras framework using Theano as a backend. The entire model is implemented as a single computation graph in order to train the model via a direct minimization of the Mean Square Error (MSE) between the system estimation and the output voltage $V_{\text {out }}$ measured. The training procedure is performed with the Gradient Descent Back Propagation algorithm considering the Nadam optimizer [66] with 2000 epoques. All the NNs have been initialized with the Glorot Normal initializer with the exception of the FLNN whose weights have been initially set to zero (for further details about the NNs activation function and optimization algorithms, a complete explanation can be found in [67]).

The FLNN activation functions are defined according to Bernstein and trigonometric polynomials in order to better fit the OCV curve [46]. Specifically, 21 neurons evaluate the Bernstein polynomials from zero to the 20th degree, whilst 20 neurons are designed as sinusoidal and cosine polynomials from the 1st to the 10th degree. The neuron on the output layer is equipped with a common sigmoid activation function. The MLP architectures instead use ReLu activation functions for the neurons in the hidden layer and sigmoid activation for the output layer. The system configuration (NNs type, input tuple, activation functions and number of neurons of hidden/output layers) has been reported in Table 3 according to the results obtained in [46].

Table 3. PI-ENNC and ENNC settings.

\begin{tabular}{|c|c|c|c|c|c|c|}
\hline \multirow{2}{*}{$\begin{array}{l}\text { Model } \\
\text { Name }\end{array}$} & \multicolumn{4}{|c|}{ ENNC \& PI-ENNC } & \multicolumn{2}{|c|}{ PI-ENNC } \\
\hline & $\mathcal{C}_{q s t}$ & $\mathcal{R}_{\text {ist }}$ & $\mathcal{R}_{d y n}$ & $\tau_{d y n}$ & $\mathcal{R}_{q s t}$ & $\tau_{q s t}$ \\
\hline Type & FLNN & MLP & MLP & MLP & MLP & MLP \\
\hline Input Tuple & $\left(S o C+S_{o} C_{d y n}\right) \mid T_{i n}$ & $I_{\text {in }}\left|S_{o C}\right| T_{\text {in }}$ & $I_{\text {in }}|\operatorname{SoC}| T_{\text {in }}$ & $I_{\text {in }}|\operatorname{SoC}| T_{\text {in }}$ & $I_{\text {in }}$ & $I_{\text {in }}$ \\
\hline \# Hidden Layers & 1 & 1 & 1 & 1 & 1 & 1 \\
\hline \# Hidden Neurons & $21+20$ & 15 & 15 & 15 & 15 & 15 \\
\hline Hidden Activation & $\{$ bern $\}+\{$ trig $\}$ & $\{R e L u\}$ & $\{R e L u\}$ & $\{R e L u\}$ & $\{R e L u\}$ & $\{\operatorname{ReLu}\}$ \\
\hline \# Output Neurons & 1 & 1 & 1 & 1 & 1 & 1 \\
\hline Output Activation & $\{s i g\}$ & $\{s i g\}$ & $\{s i g\}$ & $\{s i g\}$ & $\{s i g\}$ & $\{s i g\}$ \\
\hline
\end{tabular}

As can be observed, the PI-ENNC is featured by 122 neurons against the 90 of the ENNC. Therefore, considering the study reported in [46], the increase in the computational time can be considered irrelevant. Indeed, as mentioned in [46], in this BMS paradigm, the SR-UKF is by far the most time-consuming component making the ENNC computational cost negligible.

In order to better study the effectiveness of our model, the tests have been repeated by considering every possible combination of the input array, namely $T_{i n}, S o C$ and $I_{i n}$. It is clear that for the dataset of the NR 18650-20R cell, which does not have the $T_{i n}$ time series (see Section 6.3) the number of tests is limited to three cases.

The training phase has been conducted according to two different procedures:

- 1-Phase: all NNs blocks are trained together on the training set in a single step.

- 2-Phases: the FLNN is trained on the OCV curve in a first stage. After that, the remaining NNs can start their training phase.

The model performance is evaluated considering the MSE between the BMS SoC approximation and its real value in the test set. In order to neglect the KF transient time (the SR-UKF needs a stabilization time since it starts to work in a non-stationary working point), an offset of $30 \mathrm{~min}$ has been set with respect to the first sample of the sequence. The SR-UKF has been initialized with $S_{0} C_{0}=0.5, V_{d y n, 0}=0$ for both ENNC and PI-ENNC models and the $S o C_{d y n, 0}=0$ for PI-ENNC model. 


\section{Results}

The computational results in Table 4 show the proposed model (PI-ENNC) performance attained in terms of MSE with respect to ENNC (the best solution for each row are marked in bold). Specifically, each model has been trained according to both the 1-Phase and 2-Phases procedures. Additionally, the results are shown with different combinations of the input parameters $I_{i n}, S o C$ and $T_{i n}$. That is, different possible situations are considered where specific physical quantities are not taken into account for determining the behavior of the system. For the sake of clarity, the best configurations arise from Table 4 are graphically compared in Figure 17. Furthermore, in Figure 18, the results are written in terms of mean MSE value obtained by averaging all the possible input combinations. The same results are collected in Table 5 along with their standard deviations.

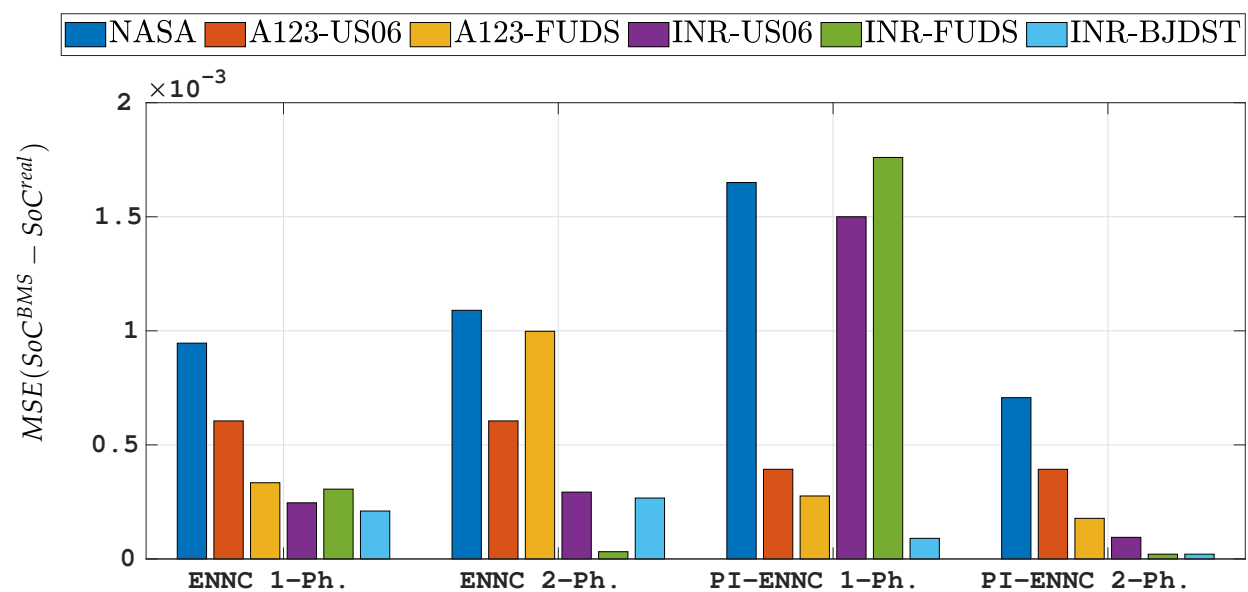

Figure 17. Best ENNC and PI-ENNC models performance on the test sets considering both training procedures, 1-Phase and 2-Phases.

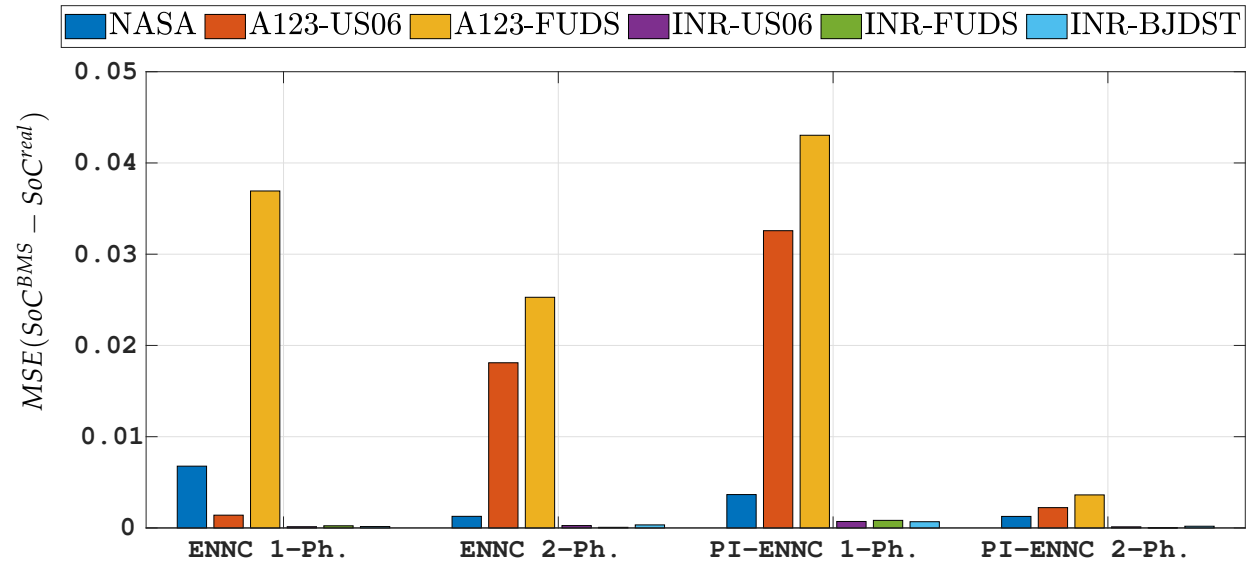

Figure 18. SoC MSE mean values considering results of Table 4 grouped per BMS prediction model and training procedure.

For the sake of completeness, in Appendix A are reported Table 4 rewritten in percentage (Table A1) and the time series of the predicted $\mathrm{SoC}$ and the output voltage referring to the PI-ENNC BMS with respect to their real values (Figures A1-A6). 
Table 4. Test results on SoC MSE sorted per dataset, BMS model, training procedure and input tuple definition.

\begin{tabular}{|c|c|c|c|c|c|c|c|c|c|}
\hline \multirow{2}{*}{$\begin{array}{l}\text { Dataset } \\
\text { NASA }\end{array}$} & \multirow{2}{*}{$\begin{array}{l}\text { Model } \\
\text { ENNC }\end{array}$} & \multirow{2}{*}{$\begin{array}{c}\text { Train. Proc. } \\
\text { 1-Phase }\end{array}$} & \multicolumn{7}{|c|}{ SoC Approximation-MSE $\left(S o C^{B M S}-S o C^{r e a l}\right)$} \\
\hline & & & $\begin{array}{c}5.01 \\
\times 10^{-3}\end{array}$ & $1.44 \times 10^{-3}$ & $1.73 \times 10^{-3}$ & $1.07 \times 10^{-3}$ & $2.01 \times 10^{-2}$ & $9.46 \times 10^{-4}$ & $1.71 \times 10^{-2}$ \\
\hline & ENNC & 2-Phases & $1.40 \times 10^{-3}$ & $1.24 \times 10^{-3}$ & $1.44 \times 10^{-3}$ & $1.28 \times 10^{-3}$ & $1.27 \times 10^{-3}$ & $1.18 \times 10^{-3}$ & $1.09 \times 10^{-3}$ \\
\hline & PI-ENNC & 1-Phase & $1.65 \times 10^{-3}$ & $2.23 \times 10^{-3}$ & $1.97 \times 10^{-3}$ & $2.64 \times 10^{-3}$ & $8.74 \times 10^{-3}$ & $3.07 \times 10^{-3}$ & $5.30 \times 10^{-3}$ \\
\hline & PI-ENNC & 2-Phases & $1.37 \times 10^{-3}$ & $1.58 \times 10^{-3}$ & $1.52 \times 10^{-3}$ & $1.36 \times 10^{-3}$ & $7.07 \times 10^{-4}$ & $1.15 \times 10^{-3}$ & $1.15 \times 10^{-3}$ \\
\hline & & & Iin & SoC & In,SoC & Tin & Tin, In & Tin,SoC & Tin,Iin,SoC \\
\hline \multicolumn{10}{|l|}{ A123 } \\
\hline \multirow[t]{4}{*}{ US06 } & ENNC & 1-Phase & $7.76 \times 10^{-4}$ & $6.05 \times 10^{-4}$ & $5.00 \times 10^{-4}$ & $2.75 \times 10^{-3}$ & $2.60 \times 10^{-3}$ & $1.13 \times 10^{-3}$ & $1.44 \times 10^{-3}$ \\
\hline & ENNC & 2-Phases & $8.12 \times 10^{-4}$ & $1.66 \times 10^{-2}$ & $5.19 \times 10^{-3}$ & $4.62 \times 10^{-3}$ & $2.29 \times 10^{-3}$ & $8.64 \times 10^{-2}$ & $1.08 \times 10^{-2}$ \\
\hline & PI-ENNC & 1-Phase & $4.36 \times 10^{-3}$ & $4.97 \times 10^{-4}$ & $3.93 \times 10^{-4}$ & $9.39 \times 10^{-2}$ & $5.96 \times 10^{-2}$ & $6.88 \times 10^{-2}$ & $4.83 \times 10^{-4}$ \\
\hline & PI-ENNC & 2-Phases & $4.96 \times 10^{-4}$ & $1.24 \times 10^{-3}$ & $2.53 \times 10^{-3}$ & $5.83 \times 10^{-4}$ & $3.41 \times 10^{-4}$ & $8.09 \times 10^{-3}$ & $2.30 \times 10^{-3}$ \\
\hline \multirow[t]{5}{*}{ FUDS } & ENNC & 1-Phase & $6.18 \times 10^{-4}$ & $3.34 \times 10^{-4}$ & $8.10 \times 10^{-4}$ & $2.26 \times 10^{-3}$ & $2.36 \times 10^{-3}$ & $2.12 \times 10^{-3}$ & $2.50 \times 10^{-1}$ \\
\hline & ENNC & 2-Phases & $9.98 \times 10^{-4}$ & $6.86 \times 10^{-3}$ & $2.95 \times 10^{-2}$ & $2.96 \times 10^{-3}$ & $2.03 \times 10^{-3}$ & $1.18 \times 10^{-1}$ & $1.66 \times 10^{-2}$ \\
\hline & PI-ENNC & 1-Phase & $2.76 \times 10^{-4}$ & $3.28 \times 10^{-4}$ & $3.70 \times 10^{-4}$ & $1.06 \times 10^{-1}$ & $9.58 \times 10^{-2}$ & $9.79 \times 10^{-2}$ & $5.70 \times 10^{-4}$ \\
\hline & PI-ENNC & 2-Phases & $1.78 \times 10^{-4}$ & $3.19 \times 10^{-3}$ & $8.09 \times 10^{-3}$ & $1.36 \times 10^{-3}$ & $1.50 \times 10^{-3}$ & $4.62 \times 10^{-3}$ & $6.40 \times 10^{-3}$ \\
\hline & & & Iin & SoC & In,SoC & Tin & Tin, In & Tin,SoC & Tin,Iin,SoC \\
\hline \multicolumn{10}{|c|}{ INR 18650-20R } \\
\hline \multirow[t]{4}{*}{ US06 } & ENNC & 1-Phase & $4.16 \times 10^{-4}$ & $2.71 \times 10^{-4}$ & $2.46 \times 10^{-4}$ & & & & \\
\hline & ENNC & 2-Phases & $6.78 \times 10^{-4}$ & $2.93 \times 10^{-4}$ & $8.31 \times 10^{-4}$ & & & & \\
\hline & PI-ENNC & 1-Phase & $1.65 \times 10^{-3}$ & $1.81 \times 10^{-3}$ & $1.50 \times 10^{-3}$ & & & & \\
\hline & PI-ENNC & 2-Phases & $5.16 \times 10^{-4}$ & $9.47 \times 10^{-5}$ & $2.26 \times 10^{-4}$ & & & & \\
\hline \multirow[t]{4}{*}{ FUDS } & ENNC & 1-Phase & $8.43 \times 10^{-4}$ & $3.06 \times 10^{-4}$ & $5.02 \times 10^{-4}$ & & & & \\
\hline & ENNC & 2-Phases & $2.04 \times 10^{-4}$ & $2.39 \times 10^{-4}$ & $3.17 \times 10^{-5}$ & & & & \\
\hline & PI-ENNC & 1-Phase & $2.22 \times 10^{-3}$ & $1.76 \times 10^{-3}$ & $1.83 \times 10^{-3}$ & & & & \\
\hline & PI-ENNC & 2-Phases & $2.09 \times 10^{-5}$ & $5.28 \times 10^{-5}$ & $3.58 \times 10^{-5}$ & & & & \\
\hline \multirow[t]{5}{*}{ BJDST } & ENNC & 1-Phase & $5.20 \times 10^{-4}$ & $2.10 \times 10^{-4}$ & $3.30 \times 10^{-4}$ & & & & \\
\hline & ENNC & 2-Phases & $8.80 \times 10^{-4}$ & $2.67 \times 10^{-4}$ & $1.18 \times 10^{-3}$ & & & & \\
\hline & PI-ENNC & 1-Phase & $1.62 \times 10^{-3}$ & $1.75 \times 10^{-3}$ & $1.40 \times 10^{-3}$ & & & & \\
\hline & PI-ENNC & 2-Phases & $9.05 \times 10^{-4}$ & $9.03 \times 10^{-5}$ & $3.11 \times 10^{-4}$ & & & & \\
\hline & & & Iin & SoC & In,SoC & & & & \\
\hline
\end{tabular}

Table 5. Mean and standard deviation on SoC MSE prediction. Results are sorted per test sets, BMS model and training procedure.

NASA

A123

\begin{tabular}{ccccccc}
\hline Dataset & \multicolumn{2}{c}{ Std.Dev. } & Mean & Std.Dev. & Mean & Std.Dev. \\
Model and tr. Procedure & Mean & Std PUD \\
ENNC 1 Phase & 0.00677 & 0.00824 & 0.00140 & 0.00093 & 0.03693 & 0.09396 \\
ENNC 2 Phases & 0.00127 & 0.00012 & 0.01810 & 0.03060 & 0.02528 & 0.04215 \\
PI-ENNC 1 Phase & 0.00366 & 0.00254 & 0.03258 & 0.04020 & 0.04303 & 0.05328 \\
PI-ENNC 2 Phases & 0.00126 & 0.00029 & 0.00223 & 0.00273 & 0.00362 & 0.00290 \\
\hline \multicolumn{7}{c}{ INR 18650-20R } \\
Dataset & \multicolumn{2}{c}{ US06 } & \multicolumn{2}{c}{ FUDS } & \multicolumn{2}{c}{ BJDTS } \\
Model and tr. Procedure & Mean & Std.Dev. & Mean & Std.Dev. & Mean & Std.Dev. \\
\hline ENNC 1 Phase & 0.00013 & 0.00017 & 0.00024 & 0.00033 & 0.00015 & 0.00021 \\
ENNC 2 Phases & 0.00026 & 0.00036 & 0.00007 & 0.00011 & 0.00033 & 0.00049 \\
PI-ENNC 1 Phase & 0.00071 & 0.00089 & 0.00083 & 0.00105 & 0.00068 & 0.00086 \\
PI-ENNC 2 Phases & 0.00012 & 0.00019 & 0.00002 & 0.00002 & 0.00019 & 0.00034 \\
\hline
\end{tabular}

By inspecting Figure 17, PI-ENNC trained in two phases emerges as the best solution. Indeed, the procedure outperforms ENNC in both 1-Phase and 2-Phases configurations in terms of MSE for all the investigated datasets. Conversely, 1-Phase PI-ENNC does not provide enough accuracy in the description of the system behavior since for NASA, 
INR-US06 and INR-FUDS the MSE values are worst with respect to both ENNC 1-Phase and 2-Phases. Figure 18 and Table 5 confirm the validity of the proposed method: the best results by means of average MSE are obtained by the PI-ENNC trained in two phases, which outperforms the competitors in all the datasets with the exception of BJDST, where the ENNC 1-Phase shows a slightly lower mean MSE (0.00019 for PI-ENNC against 0.00015 obtained by ENNC). Similar considerations hold in terms of model robustness. By comparing the best two models by means of average MSE according to the input configurations (i.e., 2-Phases PI-ENNC and 1-Phase ENNC), the proposed method shows lower standard deviations values in A123-FUDS, INR-FUDS and NASA whereas for the remaining dataset the results are comparable with ENNC trained in 1-Phase. An interesting behavior emerges by comparing the results with respect to the input $I_{i n}, T_{i n}$ and $S o C$ configuration. When the operating temperature $T_{i n}$ is considered as input (i.e., $\left[T_{i n}\right],\left[T_{i n}, I_{i n}\right]$ and $\left[T_{i n}, I_{i n}, S o C\right]$ ), the MSE attained poor performances suggesting that this parameter does not contribute to determining the complex dynamical behavior of the battery cell. Conversely, all the best results on NASA and A123 datasets are attained including the input current $I_{i n}$. Interestingly, most of the best results ( 5 out of 12) are obtained by individually considering the input current without taking into account any other parameter. Regarding the SoC input, it helps in improving BMS performance in INR 18650-20R. Indeed, only in one case (PI-ENNC trained 2-Phases simulated on FUDS) the use of the $S o C$ is ineffective, whereas in eight cases out of twelve, MSE values are more than halved with respect to solutions that considered $I_{i n}$ alone as an input parameter.

For the sake of completeness, in Table 6, the best PI-ENNC result on the NASA test set has been compared with the NN-based models discussed in [68] and the ECM optimized through an Hybrid-Genetic-PSO in [46]. The models selected for comparing the PI-ENNC are defined as follows:

- ECM: an ECM optimized through a Hybrid-Genetic-PSO in [46] whose circuital topology is the same as represented in Figure 4 of Section 4.1. The HG-PSO algorithm has been configured with 50 individuals and it has been run for 2000 iterations [46].

- $\quad$ RBF: the prediction model is defined by a Radial Basis Function (RBF) NN (black box type). It has been configured with ten hidden neurons and one output neuron with the linear activation function. The centroids of the hidden layer have been initialized with K-means clustering. The training procedure has been performed with the Nadam optimizer and trained for 10,020 epochs [68].

- ELM: an Extreme Learning Machine (ELM) [69] NN is adopted (black box type). The ELM has been set up counting ten hidden neurons with hyperbolic tangent activation function and one output neuron with linear activation. In accordance with the ELM technique, the weights of the hidden layer have been randomly initialized and the training of the output weights has been performed by solving a linear least square problem [68].

- WNN: a Wavelet Neural Network (WNN) (black box type) where the Morlet wavelet has been used as an activation function of the wavelons. K-means clustering has been used in order to initialize the translation and the dilatation parameters of the wavelets [47]. The WNN has been configured with ten hidden wavelons and one output neuron with the linear activation function. The training procedure has been performed with the Nadam optimizer and trained for 10,020 epochs [68].

All the BMS models are featured by a SR-UKF as the PI-ENNC. MSE results in Table 6 show that both PI-ENNC models are featured by lower MSE of one order of magnitude with respect to the other models.

Table 6. Comparison of the PI-ENNC SoC MSE results with the models discussed in $[46,68]$ on the NASA test set.

\begin{tabular}{ccccccc}
\hline Model & PI-ENNC & ENNC & RBF & ELM & WNN & ECM \\
\hline MSE & $7.07 \times 10^{-4}$ & $9.46 \times 10^{-4}$ & $7.916 \times 10^{-3}$ & $8.90 \times 10^{-3}$ & $7.71 \times 10^{-3}$ & $1.61 \times 10^{-3}$ \\
\hline
\end{tabular}




\title{
9. Conclusions
}

This paper proposes an extension of the ENNC BMS prediction model [46] inspired by the fractional-order ESP method studied in [34]. The extension proposed for the PI-ENNC modeling is focused on the implementation of two MLP NNs in order to approximate the effects of the SoC given by the non-uniformity of the charge in the cell due to the presence of a current load. The non-uniformity charge distribution can be approximated by a first-order low pass filter analogously to the dynamic voltage whose contribution represents the effects of the electrolyte diffusion in the presence of a load current.

The performance of the PI-ENNC model has been compared with the plain ENNC considering the accuracy of each model in predicting the battery cell SoC according to two different training procedures. Furthermore, prediction results are investigated using different combinations of the input set, namely cell current, temperature and SoC. In order to assess the validity of PI-ENNC, the tests have been conducted on three publicly available datasets properly split into training and test sets. The results witnessed the importance of designing an ENN architecture supported by proper equations and constraints describing the physical phenomenon to predict. Indeed, they show that in half the tests, the PI-ENNC outperforms ENNC in terms of MSE between the correct and the predicted SoC values, while attaining similar performances for the remaining problems. Better performance on the SoC prediction can assure different advantages on ESSs management, e.g., a better estimation of EV autonomy assuring lower commuter state of anxiety, a faster EV charging service and a better prediction of the microgrid configuration in islanded mode and autoconsumption.

In particular, the PI-ENNC model has shown promising results when the training procedure is carried out with the 2-Phases strategy, where the FLNN modeling the quasistationary contribution is trained before the other networks. Furthermore, the tests show that the operating temperature and $\mathrm{SoC}$ input can impact negatively on the system performance, highlighting instead the crucial role played by the input current in correctly estimating the system behavior.

Further studies can address the adaptation of the PI-ENNC and its corresponding ECM to a more detailed (and complex) electrochemical model, also applying a fair comparison with other affirmed methods proposed in the literature.

Author Contributions: Conceptualization, S.L.; methodology, S.L.; software, S.L.; validation, S.L.; formal analysis, S.L.; investigation, S.L.; resources, A.R. and F.M.F.M.; data curation, S.L.; writingoriginal draft preparation, S.L. and L.B.; writing-review and editing, S.L. and L.B.; visualization, S.L.; supervision, S.L. and F.M.F.M.; project administration, S.L.; funding acquisition, F.M.F.M. All authors have read and agreed to the published version of the manuscript.

Funding: This research received no external funding.

Institutional Review Board Statement: Not applicable.

Informed Consent Statement: Not applicable.

Acknowledgments: The authors want to thank Massimiliano Luzi for his previous works that initiated our team on the modeling techniques discussed throughout this paper and Gabriele Perri who contributed to the implementation.

Conflicts of Interest: The authors declare no conflict of interest.

\author{
Abbreviations \\ The following abbreviations are used in this manuscript: \\ BMS Battery Management System \\ CC Coulomb Counting \\ DST Dynamic Stress Test \\ ECM Equivalent Circuit Model \\ EIS Electrochemical Impedance Spectroscopy
}




$\begin{array}{ll}\text { EKF } & \text { Extended Kalman Filter } \\ \text { ELM } & \text { Extreme Learning Machine } \\ \text { ENNC } & \text { Equivalent Neural Network Circuit } \\ \text { ESP } & \text { Extended Single Particle } \\ \text { ESS } & \text { Energy Storage System } \\ \text { EV } & \text { Electric Vehicle } \\ \text { FIS } & \text { Fuzzy Inference System } \\ \text { FLNN } & \text { Functional Link Neural Network } \\ \text { FUDS } & \text { Federal Urban Driving Schedule } \\ \text { KF } & \text { Kalman Filter } \\ \text { MLP } & \text { Multi Layer Perceptron } \\ \text { MSE } & \text { Mean Square Error } \\ \text { NN } & \text { Neural Network } \\ \text { OCV } & \text { Open Circuit Voltage } \\ \text { PI-ENNC } & \text { Physical Inspired-Equivalent Neural Network Circuit } \\ \text { PSO } & \text { Particle Swarm Optimization } \\ \text { P2D } & \text { Pseudo-two-Dimensional } \\ \text { RBF } & \text { Radial Basis Function } \\ \text { RNN } & \text { Recurrent Neural Network } \\ \text { SoC } & \text { State of Charge } \\ \text { SR-UKF } & \text { Square Root Unscented Kalman Filter } \\ \text { SVM } & \text { Support Vector Machine } \\ \text { UKF } & \text { Unscented Kalman Filter } \\ \text { WNN } & \text { Wavelet Neural Network } \\ \text { V2G } & \text { Vehicle to Grid }\end{array}$

\section{Appendix A}

The following are illustrations of some extra results regarding the best performance of the PI-ENNC model. Specifically, Figure A1 presents plots of the SoC time series of the PI-ENNC model and the ENNC model compared with the reference SoC time series to approximate; in Figures A2-A6, the output voltage and the SoC time series generated on the best PI-ENNC model on the A123 and INR 18650-20R test sets are shown. In Table A1, the test results of Table 4 are reported in percentages.

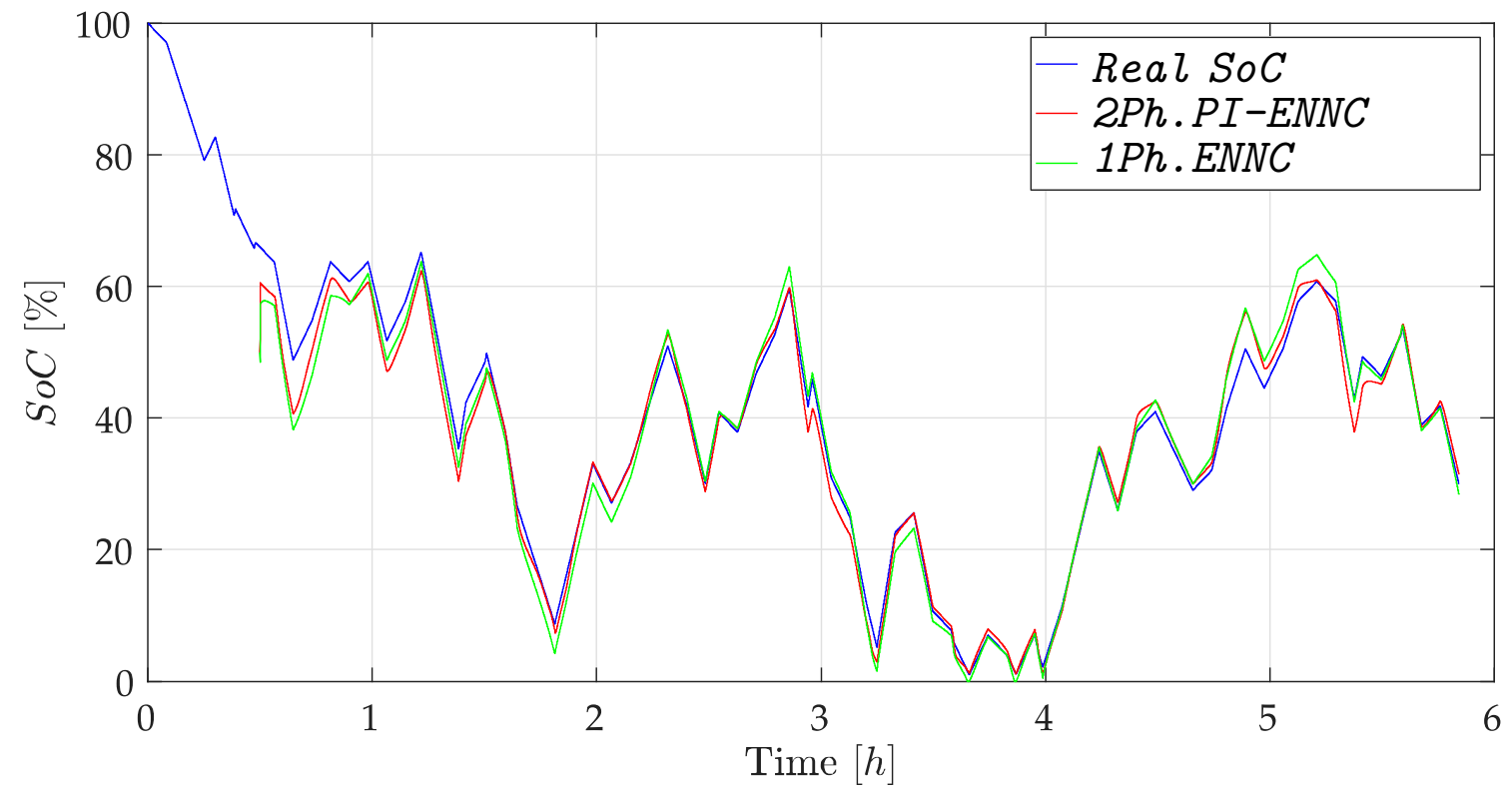

Figure A1. Comparison of the ENNC and PI-ENNC SoC prediction time series on the test set referring to the NASA dataset. 

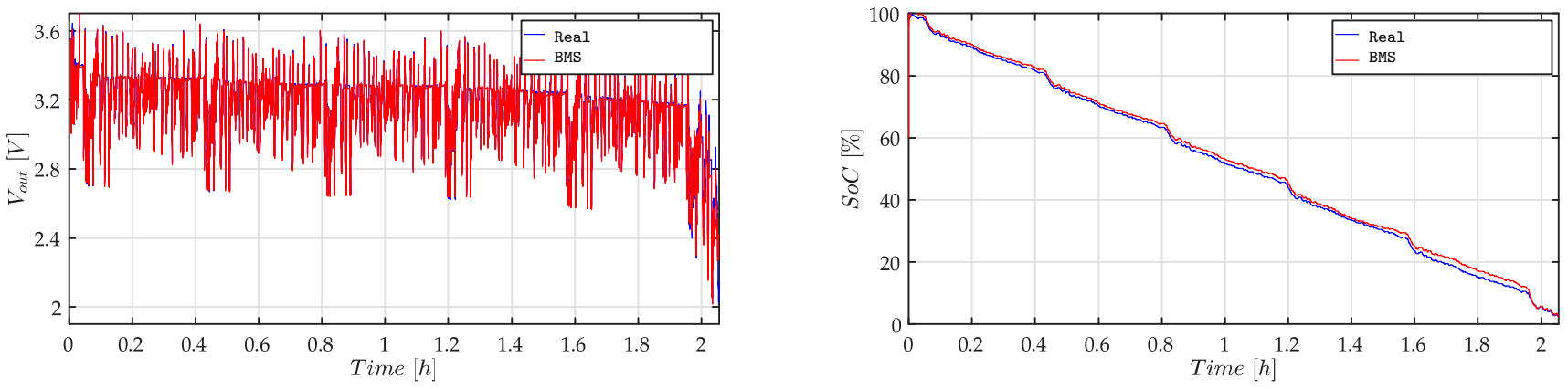

Figure A2. SoC and $V_{\text {out }}$ time series referring to the A123-FUDS dataset considering the best PI-ENNC BMS model on the test set.
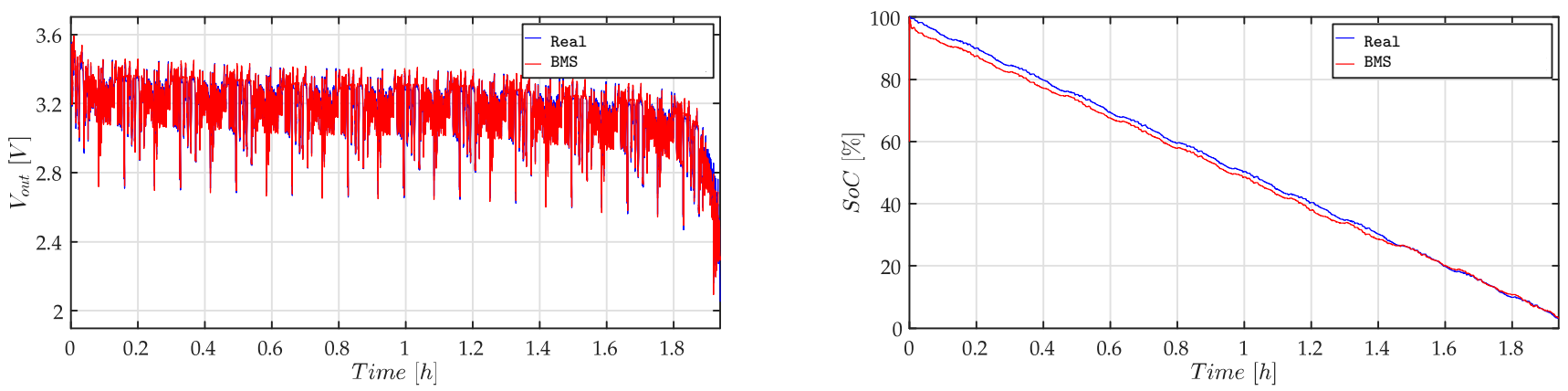

Figure A3. SoC and $V_{\text {out }}$ time series referring to the A123-US06 dataset considering the best PI-ENNC BMS model on the test set.
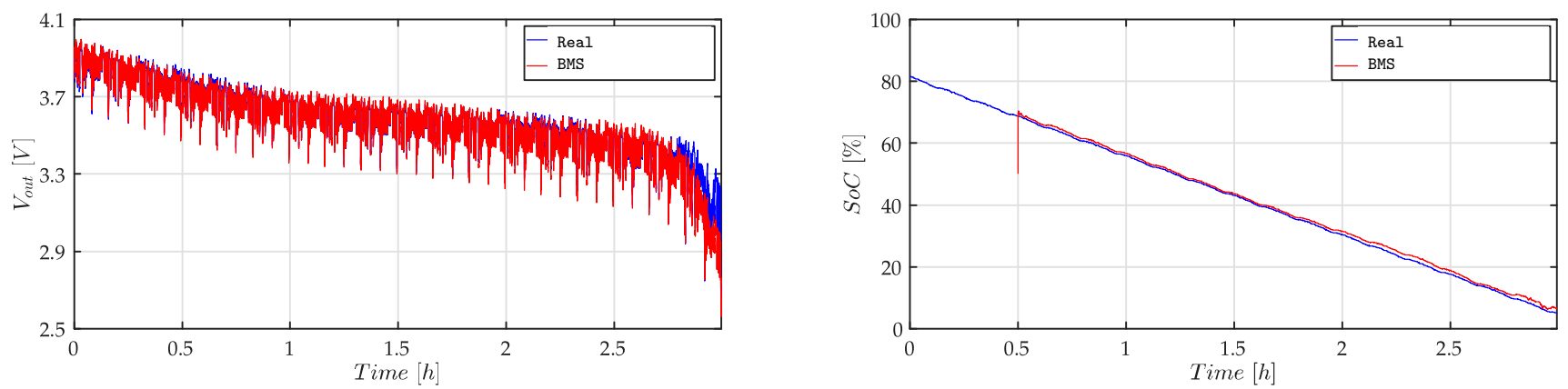

Figure A4. SoC and $V_{\text {out }}$ time series referring to the INR 18650-20R-US06 dataset considering the best PI-ENNC BMS model on the test set.
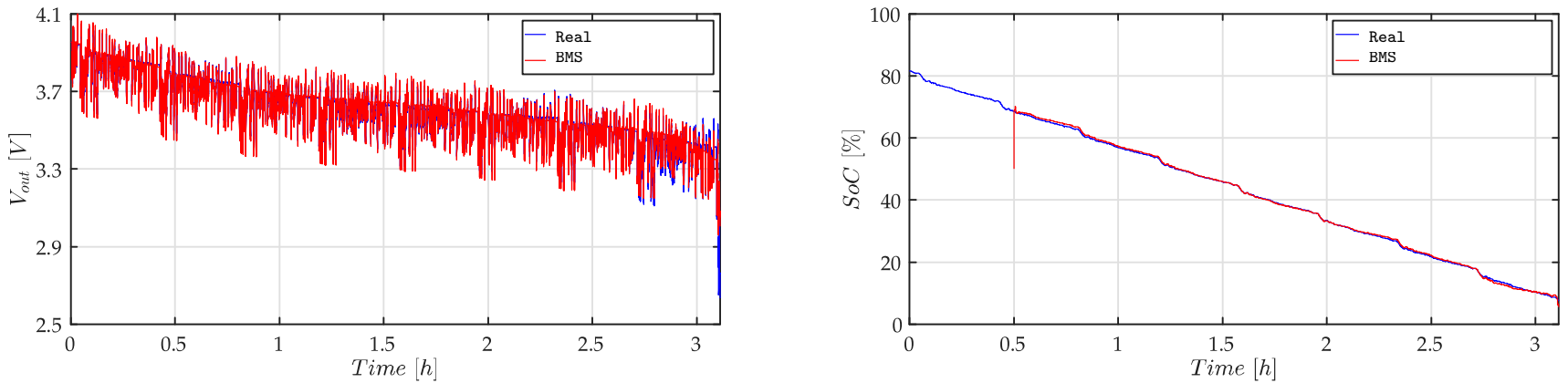

Figure A5. SoC and $V_{\text {out }}$ time series referring to the INR 18650-20R-FUDS dataset considering the best PI-ENNC BMS model on the test set. 

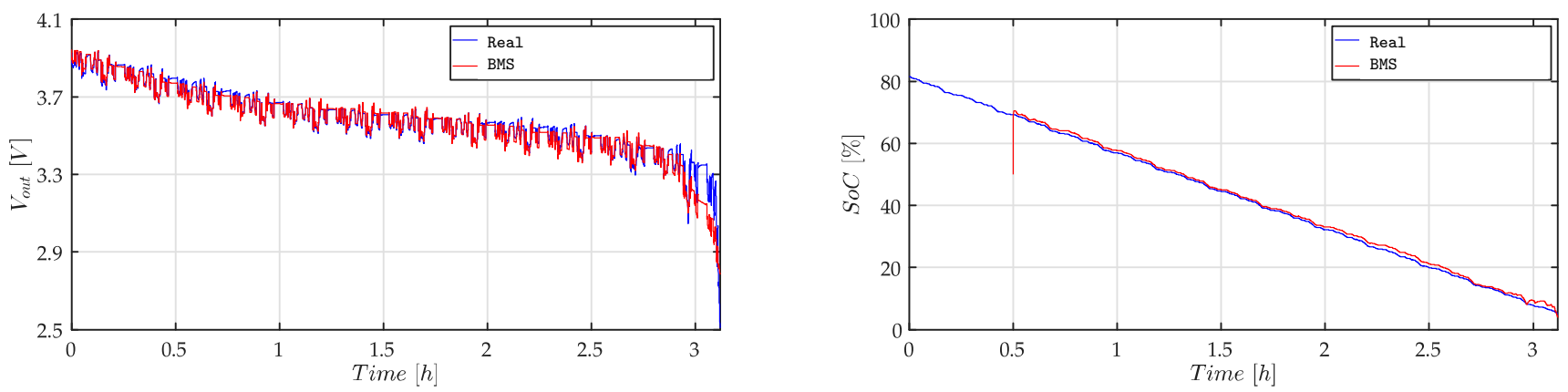

Figure A6. SoC and $V_{\text {out }}$ time series referring to the INR 18650-20R-BJDST dataset considering the best PI-ENNC BMS model on the test set.

Table A1. Test result on SoC MSE (in percent) sorted per dataset, BMS model, training procedure and input tuple definition.

\begin{tabular}{|c|c|c|c|c|c|c|c|c|c|}
\hline \multirow{2}{*}{$\frac{\text { Dataset }}{\text { NASA }}$} & \multirow{2}{*}{$\begin{array}{l}\text { Model } \\
\text { ENNC }\end{array}$} & \multicolumn{2}{|l|}{ Train. Proc. } & \multicolumn{5}{|c|}{ SoC Approximation-MSE $\left(S o C^{B M S}-S o C^{r e a l}\right)$} & \multirow[b]{2}{*}{$1.71 \%$} \\
\hline & & 1-Phase & $0.50 \%$ & $0.14 \%$ & $0.17 \%$ & $0.10 \%$ & $2.01 \%$ & $0.09 \%$ & \\
\hline & ENNC & 2-Phases & $0.14 \%$ & $0.12 \%$ & $0.14 \%$ & $0.13 \%$ & $0.13 \%$ & $0.12 \%$ & $0.11 \%$ \\
\hline & PI-ENNC & 1-Phase & $0.16 \%$ & $0.22 \%$ & $0.20 \%$ & $0.26 \%$ & $0.87 \%$ & $0.31 \%$ & $0.53 \%$ \\
\hline & PI-ENNC & 2-Phases & $0.14 \%$ & $0.16 \%$ & $0.15 \%$ & $0.14 \%$ & $0.07 \%$ & $0.11 \%$ & $0.11 \%$ \\
\hline & & & Iin & SoC & In,SoC & Tin & Tin, In & Tin,SoC & Tin,Iin,SoC \\
\hline \multicolumn{10}{|l|}{ A123 } \\
\hline \multirow[t]{4}{*}{ US06 } & ENNC & 1-Phase & $0.07 \%$ & $0.06 \%$ & $0.05 \%$ & $0.27 \%$ & $0.26 \%$ & $0.11 \%$ & $0.14 \%$ \\
\hline & ENNC & 2-Phases & $0.08 \%$ & $1.66 \%$ & $0.52 \%$ & $0.46 \%$ & $0.23 \%$ & $8.64 \%$ & $1.08 \%$ \\
\hline & PI-ENNC & 1-Phase & $0.44 \%$ & $0.50 \%$ & $0.39 \%$ & $9.39 \%$ & $5.96 \%$ & $6.88 v$ & $0.04 \%$ \\
\hline & PI-ENNC & 2-Phases & $0.05 \%$ & $0.12 \%$ & $0.25 \%$ & $0.06 \%$ & $0.03 \%$ & $0.81 \%$ & $0.23 \%$ \\
\hline \multirow[t]{5}{*}{ FUDS } & ENNC & 1-Phase & $0.06 \%$ & $0.03 \%$ & $0.08 \%$ & $0.22 \%$ & $0.24 \%$ & $0.21 \%$ & $25 \%$ \\
\hline & ENNC & 2-Phases & $0.09 \%$ & $0.69 \%$ & $2.95 \%$ & $0.30 \%$ & $0.20 \%$ & $11.8 \%$ & $1.66 \%$ \\
\hline & PI-ENNC & 1-Phase & $0.03 \%$ & $0.033 \%$ & $0.037 \%$ & $11 \%$ & $9.58 \%$ & $9.79 \%$ & $0.06 \%$ \\
\hline & PI-ENNC & 2-Phases & $0.02 \%$ & $0.32 \%$ & $0.81 \%$ & $0.14 \%$ & $0.15 \%$ & $0.46 \%$ & $0.64 \%$ \\
\hline & & & Iin & SoC & In,SoC & Tin & Tin, In & Tin,SoC & Tin,Iin,SoC \\
\hline \multicolumn{10}{|c|}{ INR 18650-20R } \\
\hline \multirow[t]{4}{*}{ US06 } & ENNC & 1-Phase & $0.04 \%$ & $0.03 \%$ & $0.02 \%$ & & & & \\
\hline & ENNC & 2-Phases & $0.07 \%$ & $0.03 \%$ & $0.08 \%$ & & & & \\
\hline & PI-ENNC & 1-Phase & $0.16 \mathrm{e}-03$ & $0.18 \%$ & $0.15 \%$ & & & & \\
\hline & PI-ENNC & 2-Phases & $0.052 \%$ & $0.009 \%$ & $0.02 \%$ & & & & \\
\hline \multirow[t]{4}{*}{ FUDS } & ENNC & 1-Phase & $0.08 \%$ & $0.03 \%$ & $0.05 \%$ & & & & \\
\hline & ENNC & 2-Phases & $0.02 \%$ & $0.02 \%$ & $0.003 \%$ & & & & \\
\hline & PI-ENNC & 1-Phase & $0.22 \%$ & $0.18 \%$ & $0.18 \%$ & & & & \\
\hline & PI-ENNC & 2-Phases & $0.002 \%$ & $0.005 \%$ & $0.003 \%$ & & & & \\
\hline \multirow[t]{5}{*}{ BJDST } & ENNC & 1-Phase & $0.05 \%$ & $0.02 \%$ & $0.03 \%$ & & & & \\
\hline & ENNC & 2-Phases & $0.09 \%$ & $0.03 \%$ & $0.12 \%$ & & & & \\
\hline & PI-ENNC & 1-Phase & $0.16 \%$ & $0.17 \%$ & $0.14 \%$ & & & & \\
\hline & PI-ENNC & 2-Phases & $0.09 \%$ & $0.009 \%$ & $0.031 \%$ & & & & \\
\hline & & & Iin & SoC & In,SoC & & & & \\
\hline
\end{tabular}

\section{References}

1. Osório, G.J.; Shafie-khah, M.; Carvalho, G.C.R.; Catalão, J.P.S. Analysis Application of Controllable Load Appliances Management in a Smart Home. Energies 2019, 12, 3710. [CrossRef]

2. Bibak, B.; Tekiner-Moğulkoç, H. A comprehensive analysis of Vehicle to Grid (V2G) systems and scholarly literature on the application of such systems. Renew. Energy Focus 2021, 36, 1-20. [CrossRef]

3. ur Rehman, U.; Riaz, M.; Wani, M.Y. A robust optimization method for optimizing day-ahead operation of the electric vehicles aggregator. Int. J. Electr. Power Energy Syst. 2021, 132, 107179. [CrossRef]

4. Leonori, S.; Rizzoni, G.; Frattale Mascioli, F.M.; Rizzi, A. Intelligent energy flow management of a nanogrid fast charging station equipped with second life batteries. Int. J. Electr. Power Energy Syst. 2021, 127, 106602. [CrossRef] 
5. Hannan, M.A.; Hoque, M.M.; Hussain, A.; Yusof, Y.; Ker, P.J. State-of-the-Art and Energy Management System of Lithium-Ion Batteries in Electric Vehicle Applications: Issues and Recommendations. IEEE Access 2018, 6, 19362-19378. [CrossRef]

6. Duffner, F.; Wentker, M.; Greenwood, M.; Leker, J. Battery cost modeling: A review and directions for future research. Renew. Sustain. Energy Rev. 2020, 127, 109872. [CrossRef]

7. Lelie, M.; Braun, T.; Knips, M.; Nordmann, H.; Ringbeck, F.; Zappen, H.; Sauer, D.U. Battery Management System Hardware Concepts: An Overview. Appl. Sci. 2018, 8, 534. [CrossRef]

8. Rahimi-Eichi, H.; Ojha, U.; Baronti, F.; Chow, M.Y. Battery Management System: An Overview of Its Application in the Smart Grid and Electric Vehicles. IEEE Ind. Electron. Mag. 2013, 7, 4-16. [CrossRef]

9. Dai, H.; Jiang, B.; Hu, X.; Lin, X.; Wei, X.; Pecht, M. Advanced battery management strategies for a sustainable energy future: Multilayer design concepts and research trends. Renew. Sustain. Energy Rev. 2021, 138, 110480. [CrossRef]

10. Hossain Lipu, M.; Hannan, M.; Karim, T.F.; Hussain, A.; Saad, M.H.M.; Ayob, A.; Miah, M.S.; Indra Mahlia, T. Intelligent algorithms and control strategies for battery management system in electric vehicles: Progress, challenges and future outlook. J. Clean. Prod. 2021, 292, 126044. [CrossRef]

11. Ali, M.U.; Zafar, A.; Nengroo, S.H.; Hussain, S.; Junaid Alvi, M.; Kim, H.J. Towards a Smarter Battery Management System for Electric Vehicle Applications: A Critical Review of Lithium-Ion Battery State of Charge Estimation. Energies 2019, 12, 446. [CrossRef]

12. Chaturvedi, N.A.; Klein, R.; Christensen, J.; Ahmed, J.; Kojic, A. Algorithms for Advanced Battery-Management Systems. IEEE Control. Syst. Mag. 2010, 30, 49-68. [CrossRef]

13. Rivera-Barrera, J.P.; Muñoz-Galeano, N.; Sarmiento-Maldonado, H.O. SoC Estimation for Lithium-ion Batteries: Review and Future Challenges. Electronics 2017, 6, 102. [CrossRef]

14. Ng, K.S.; Huang, Y.F.; Moo, C.S.; Hsieh, Y.C. An enhanced coulomb counting method for estimating state-of-charge and state-of-health of lead-acid batteries. In Proceedings of the INTELEC 2009-31st International Telecommunications Energy Conference, Incheon, Korea, 18-22 October 2009; pp. 1-5. [CrossRef]

15. Waag, W.; Fleischer, C.; Sauer, D.U. Critical review of the methods for monitoring of lithium-ion batteries in electric and hybrid vehicles. J. Power Sources 2014, 258, 321-339. [CrossRef]

16. Meyers, J.P.; Doyle, M.; Darling, R.M.; Newman, J. The impedance response of a porous electrode composed of intercalation particles. J. Electrochem. Soc. 2000, 147, 2930. [CrossRef]

17. Paschero, M.; Storti, G.L.; Rizzi, A.; Mascioli, F.M.F.; Rizzoni, G. A Novel Mechanical Analogy-Based Battery Model for SoC Estimation Using a Multicell EKF. IEEE Trans. Sustain. Energy 2016, 7, 1695-1702. [CrossRef]

18. Ng, K.S.; Moo, C.S.; Chen, Y.P.; Hsieh, Y.C. Enhanced coulomb counting method for estimating state-of-charge and state-of-health of lithium-ion batteries. Appl. Energy 2009, 86, 1506-1511. [CrossRef]

19. Benmouna, A.; Becherif, M.; Boulon, L.; Dépature, C.; Ramadan, H.S. Efficient experimental energy management operating for FC/battery/SC vehicles via hybrid Artificial Neural Networks-Passivity Based Control. Renew. Energy 2021, 178, 1291-1302. [CrossRef]

20. Movassagh, K.; Raihan, S.A.; Balasingam, B. Performance Analysis of Coulomb Counting Approach for State of Charge Estimation. In Proceedings of the 2019 IEEE Electrical Power and Energy Conference (EPEC), Montréal, QC, Canada, 16-18 October 2019; pp. 1-6. [CrossRef]

21. Movassagh, K.; Raihan, A.; Balasingam, B.; Pattipati, K. A Critical Look at Coulomb Counting Approach for State of Charge Estimation in Batteries. Energies 2021, 14, 4074. [CrossRef]

22. Sun, D.; Yu, X.; Wang, C.; Zhang, C.; Huang, R.; Zhou, Q.; Amietszajew, T.; Bhagat, R. State of charge estimation for lithium-ion battery based on an Intelligent Adaptive Extended Kalman Filter with improved noise estimator. Energy 2021, $214,119025$. [CrossRef]

23. Wan, E.; Van Der Merwe, R. The unscented Kalman filter for nonlinear estimation. In Proceedings of the IEEE 2000 Adaptive Systems for Signal Processing, Communications, and Control Symposium (Cat. No.00EX373), Lake Louise, AB, Canada, 1-4 October 2000; pp. 153-158. [CrossRef]

24. Khalil, I.; Ahsan, M.; Ullah, I.; Adnan, A.; Khan, N.; Nawaz, S. SOC prediction of Lithium-Ion Battery using Extended Kalman Filter. In Proceedings of the 2018 International Symposium on Recent Advances in Electrical Engineering (RAEE), Islamabad, Pakistan, 17-18 October 2018; pp. 1-5. [CrossRef]

25. Luzi, M.; Paschero, M.; Rossini, A.; Rizzi, A.; Mascioli, F.M.F. Comparison between two nonlinear Kalman Filters for reliable SoC estimation on a prototypal BMS. In Proceedings of the IECON 2016-42nd Annual Conference of the IEEE Industrial Electronics Society, Florence, Italy, 23-26 October 2016; pp. 5501-5506. [CrossRef]

26. Wei, Z.; Zhao, J.; Ji, D.; Tseng, K.J. A multi-timescale estimator for battery state of charge and capacity dual estimation based on an online identified model. Appl. Energy 2017, 204, 1264-1274. [CrossRef]

27. Charkhgard, M.; Farrokhi, M. State-of-Charge Estimation for Lithium-Ion Batteries Using Neural Networks and EKF. IEEE Trans. Ind. Electron. 2010, 57, 4178-4187. [CrossRef]

28. Espedal, I.B.; Jinasena, A.; Burheim, O.S.; Lamb, J.J. Current Trends for State-of-Charge (SoC) Estimation in Lithium-Ion Battery Electric Vehicles. Energies 2021, 14, 3284. [CrossRef]

29. Jokar, A.; Rajabloo, B.; Désilets, M.; Lacroix, M. Review of simplified Pseudo-two-Dimensional models of lithium-ion batteries. J. Power Sources 2016, 327, 44-55. [CrossRef] 
30. Doyle, M.; Fuller, T.F.; Newman, J. Modeling of Galvanostatic Charge and Discharge of the Lithium/Polymer/Insertion Cell. J. Electrochem. Soc. 1993, 140, 1526-1533. [CrossRef]

31. Wu, L.; Liu, K.; Pang, H.; Jin, J. Online SOC Estimation Based on Simplified Electrochemical Model for Lithium-Ion Batteries Considering Current Bias. Energies 2021, 14, 5265. [CrossRef]

32. Gao, Y.; Zhu, C.; Zhang, X.; Guo, B. Implementation and evaluation of a practical electrochemical- thermal model of lithium-ion batteries for EV battery management system. Energy 2021, 221, 119688. [CrossRef]

33. Kemper, P.; Li, S.E.; Kum, D. Simplification of pseudo two dimensional battery model using dynamic profile of lithium concentration. J. Power Sources 2015, 286, 510-525. [CrossRef]

34. Li, X.; Fan, G.; Pan, K.; Wei, G.; Zhu, C.; Rizzoni, G.; Canova, M. A physics-based fractional order model and state of energy estimation for lithium ion batteries. Part I: Model development and observability analysis. J. Power Sources 2017, 367, 187-201. [CrossRef]

35. Li, X.; Pan, K.; Fan, G.; Lu, R.; Zhu, C.; Rizzoni, G.; Canova, M. A physics-based fractional order model and state of energy estimation for lithium ion batteries. Part II: Parameter identification and state of energy estimation for LiFePO4 battery. J. Power Sources 2017, 367, 202-213. [CrossRef]

36. Chen, L.; Wang, Z.; Lü, Z.; Li, J.; Ji, B.; Wei, H.; Pan, H. A Novel State-of-Charge Estimation Method of Lithium-Ion Batteries Combining the Grey Model and Genetic Algorithms. IEEE Trans. Power Electron. 2018, 33, 8797-8807. [CrossRef]

37. Yu, Z.; Xiao, L.; Li, H.; Zhu, X.; Huai, R. Model Parameter Identification for Lithium Batteries Using the Coevolutionary Particle Swarm Optimization Method. IEEE Trans. Ind. Electron. 2017, 64, 5690-5700. [CrossRef]

38. Hu, X.; Li, S.; Peng, H. A comparative study of equivalent circuit models for Li-ion batteries. J. Power Sources 2012, 198, 359-367. [CrossRef]

39. Zhou, S.; Liu, X.; Hua, Y.; Zhou, X.; Yang, S. Adaptive model parameter identification for lithium-ion batteries based on improved coupling hybrid adaptive particle swarm optimization- simulated annealing method. J. Power Sources 2021, $482,228951$. [CrossRef]

40. Luzi, M.; Paschero, M.; Rizzi, A.; Frattale Mascioli, F.M. A PSO algorithm for transient dynamic modeling of lithium cells through a nonlinear RC filter. In Proceedings of the 2016 IEEE Congress on Evolutionary Computation (CEC), Vancouver, BC, Canada, 24-29 July 2016; pp. 279-286. [CrossRef]

41. Dai, H.; Guo, P.; Wei, X.; Sun, Z.; Wang, J. ANFIS (adaptive neuro-fuzzy inference system) based online SOC (State of Charge) correction considering cell divergence for the EV (electric vehicle) traction batteries. Energy 2015, 80, 350-360. [CrossRef]

42. Luzi, M.; Paschero, M.; Rizzi, A.; Mascioli, F.M.F. An ANFIS Based System Identification Procedure for Modeling Electrochemical Cells. In Proceedings of the 2018 International Joint Conference on Neural Networks (IJCNN), Rio de Janeiro, Brazil, 8-13 July 2018; pp. 1-8. [CrossRef]

43. Yang, F.; Song, X.; Xu, F.; Tsui, K.L. State-of-Charge Estimation of Lithium-Ion Batteries via Long Short-Term Memory Network. IEEE Access 2019, 7, 53792-53799. [CrossRef]

44. Khalid, A.; Sundararajan, A.; Acharya, I.; Sarwat, A.I. Prediction of Li-Ion Battery State of Charge Using Multilayer Perceptron and Long Short-Term Memory Models. In Proceedings of the 2019 IEEE Transportation Electrification Conference and Expo (ITEC), Detroit, MI, USA, 19-21 June 2019; pp. 1-6. [CrossRef]

45. Ren, X.; Liu, S.; Yu, X.; Dong, X. A method for state-of-charge estimation of lithium-ion batteries based on PSO-LSTM. Energy 2021, 234, 121236. [CrossRef]

46. Luzi, M.; Frattale Mascioli, F.M.; Paschero, M.; Rizzi, A. A White-Box Equivalent Neural Network Circuit Model for SoC Estimation of Electrochemical Cells. IEEE Trans. Neural Netw. Learn. Syst. 2019, 31, 371-382. [CrossRef]

47. Dong, G.; Zhang, X.; Zhang, C.; Chen, Z. A method for state of energy estimation of lithium-ion batteries based on neural network model. Energy 2015, 90, 879-888. [CrossRef]

48. Álvarez Antón, J.; García Nieto, P.; de Cos Juez, F.; Sánchez Lasheras, F.; González Vega, M.; Roqueñí Gutiérrez, M. Battery state-of-charge estimator using the SVM technique. Appl. Math. Model. 2013, 37, 6244-6253. [CrossRef]

49. Li, J.; Ye, M.; Meng, W.; Xu, X.; Jiao, S. A Novel State of Charge Approach of Lithium Ion Battery Using Least Squares Support Vector Machine. IEEE Access 2020, 8, 195398-195410. [CrossRef]

50. Zhou, Z.H.; Chen, S. Neural network ensemble. Chin. J. Comput. Chin. Ed. 2002, 25, 1-8.

51. Zhang, J.; Xia, C. State-of-charge estimation of valve regulated lead acid battery based on multi-state Unscented Kalman Filter. Int. J. Electr. Power Energy Syst. 2011, 33, 472-476. [CrossRef]

52. Partovibakhsh, M.; Liu, G. An Adaptive Unscented Kalman Filtering Approach for Online Estimation of Model Parameters and State-of-Charge of Lithium-Ion Batteries for Autonomous Mobile Robots. IEEE Trans. Control Syst. Technol. 2015, 23, 357-363. [CrossRef]

53. Xiong, R.; Sun, F.; Chen, Z.; He, H. A data-driven multi-scale extended Kalman filtering based parameter and state estimation approach of lithium-ion polymer battery in electric vehicles. Appl. Energy 2014, 113, 463-476. [CrossRef]

54. der Merwe, R.V.; Wan, E.A. The square-root unscented Kalman filter for state and parameter-estimation. In Proceedings of the 2001 IEEE International Conference on Acoustics, Speech, and Signal Processing. Proceedings (Cat. No.01CH37221), Salt Lake City, UT, USA, 7-11 May 2001; Volume 6, pp. 3461-3464. [CrossRef] 
55. Ismail, M.; Dlyma, R.; Elrakaybi, A.; Ahmed, R.; Habibi, S. Battery state of charge estimation using an Artificial Neural Network. In Proceedings of the 2017 IEEE Transportation Electrification Conference and Expo (ITEC), Chicago, IL, USA, 22-24 June 2017; pp. 342-349. [CrossRef]

56. Chemali, E.; Kollmeyer, P.J.; Preindl, M.; Ahmed, R.; Emadi, A. Long Short-Term Memory Networks for Accurate State-of-Charge Estimation of Li-ion Batteries. IEEE Trans. Ind. Electron. 2018, 65, 6730-6739. [CrossRef]

57. Zhao, F.; Li, P.; Li, Y.; Li, Y. The Li-ion Battery State of Charge Prediction of Electric Vehicle Using Deep Neural Network. In Proceedings of the 2019 Chinese Control and Decision Conference (CCDC), Nanchang, China, 3-5 June 2019; pp. 773-777. [CrossRef]

58. Fan, G.; Pan, K.; Canova, M.; Marcicki, J.; Yang, X.G. Modeling of Li-Ion Cells for Fast Simulation of High C-Rate and Low Temperature Operations. J. Electrochem. Soc. 2016, 163, A666-A676. [CrossRef]

59. Luo, W.; Lyu, C.; Wang, L.; Zhang, L. An approximate solution for electrolyte concentration distribution in physics-based lithium-ion cell models. Microelectron. Reliab. 2013, 53, 797-804. [CrossRef]

60. Han, X.; Ouyang, M.; Lu, L.; Li, J. Simplification of physics-based electrochemical model for lithium ion battery on electric vehicle. Part II: Pseudo-two-dimensional model simplification and state of charge estimation. J. Power Sources 2015, 278, 814-825. [CrossRef]

61. Li, J.; Adewuyi, K.; Lotfi, N.; Landers, R.; Park, J. A single particle model with chemical/mechanical degradation physics for lithium ion battery State of Health (SOH) estimation. Appl. Energy 2018, 212, 1178-1190. [CrossRef]

62. dos Reis, G.; Strange, C.; Yadav, M.; Li, S. Lithium-ion battery data and where to find it. Energy AI 2021, 5, 100081. [CrossRef]

63. Kulkarni, B.B.C.; Daigle, M. Randomized Battery Usage Data Set. In NASA Ames Prognostics Data Repository; NASA Ames Research Center: Moffett Field, CA, USA, 2014.

64. Pecht, M.G. Randomized Battery Usage Data Set; Center for Advanced Life Cycle Engineering: College Park, MD, USA, 2014.

65. Zheng, F.; Xing, Y.; Jiang, J.; Sun, B.; Kim, J.; Pecht, M. Influence of different open circuit voltage tests on state of charge online estimation for lithium-ion batteries. Appl. Energy 2016, 183, 513-525. [CrossRef]

66. Dozat, T. Incorporating Nesterov Momentum into Adam. 2016. Available online: https://openreview.net/forum?id=OM0jvwB8 jIp57ZJjtNEZ\&noteId=nx924kDvKc7lP3z2iomv (accessed on 1 November 2021).

67. Aggarwal, C.C. Neural Networks and Deep Learning; Springer: Yorktown Heights, NY, USA, 2018; Volume 10, p. 497.

68. Luzi, M.; Paschero, M.; Rizzi, A.; Maiorino, E.; Frattale Mascioli, F.M. A Novel Neural Networks Ensemble Approach for Modeling Electrochemical Cells. IEEE Trans. Neural Netw. Learn. Syst. 2019, 30, 343-354. [CrossRef]

69. Huang, G.B.; Zhu, Q.Y.; Siew, C.K. Extreme learning machine: Theory and applications. Neurocomputing 2006, 70, 489-501. [CrossRef] 